\title{
Exact SINR Statistics in the Presence of Heterogeneous Interferers
}

\author{
Arman Shojaeifard, Member, IEEE, Khairi Ashour Hamdi, Senior Member, IEEE, \\ Emad Alsusa, Senior Member, IEEE, Daniel K. C. So, Senior Member, IEEE, and Jie Tang, Member, IEEE
}

\begin{abstract}
We derive new results for the higher-order moments of signal-to-interference-plus-noise ratio (SINR) in the presence of an arbitrary Poisson point process (PPP)-based heterogeneous interference field. The analysis leverages on a momentgenerating-function (MGF) methodology which only requires the statistics of intended signal and aggregate interference, thus eliminating the need for the exact distribution of SINR. We extend the existing results on interference statistics by deriving a generalized closed-form expression of the interference MGF considering Nakagami-m fading channels with exclusion region. In certain special cases, explicit expressions for the averages of different functions of SINR are found which also lead to closedform solutions for the probability distributions of aggregate interference reciprocal and signal-to-interference ratio (SIR). We prove that in such cases the effect of total PPP-based interference power on useful transmission is mathematically equivalent to the severe fluctuations from a one-sided Gaussian fading channel. As an application example, the proposed methodology is used together with stochastic geometry theory to characterize the average SINR and rate in heterogeneous cellular networks (HetNets). The validity of our analytical derivations is confirmed via Monte-Carlo simulations for various system settings. We show that with cellular network densification there exists a trade-off between the average SINR and rate performance.
\end{abstract}

Index Terms-Average performance analysis, point processes, interference characterization, higher-order moments of SINR, stochastic geometry theory, multi-tier cellular networks.

\section{INTRODUCTION}

Signal-to-interference-plus-noise ratio (SINR) is a fundamental performance measure for the design and analysis of wireless communication systems. It is defined as the ratio of the received powers from the 'useful' and the 'sum of heterogeneous interfering plus noise' signals, which are functions of the channel propagation effects such as distance-dependent path-loss, shadowing, and fading processes [1]. The knowledge of the statistics of SINR allows for the computation of other information-theoretic merits of interest such as coverage probability, spectral efficiency, and bit or symbol error rates [2]. On the other hand, the dense, irregular, and overlapping placement of nodes in emerging cellular networks renders the adoption of conventional deterministic hexagonal-grid [3] and Wyner [4] models obsolete. To this end, recently, mathematical tools from applied probability, such as stochastic geometry theory, have been utilized for deriving tractable network performance

The authors are with the Microwave and Communication Systems Group, School of Electrical and Electronic Engineering, University of Manchester, Manchester, M13 9PL, England, United Kingdom.

Email: \{a.shojaeifard,k.hamdi,e.alsusa,d.so,jie.tang\}@manchester.ac.uk

This work was supported by the Engineering and Physical Sciences Research Council (EPSRC) under grant EP/J021768/1. bounds based on the Poisson point process (PPP)-based abstraction model of interfering nodes [5], [6]. The reader is referred to [7] and the references therein for a literature survey related to the stochastic geometry-based modeling of wireless networks.

The classical approach for calculating the exact statistics of SINR requires the perfect knowledge of the probability density function (pdf) which in general is very difficult to obtain, if not impossible [8], [9]. Most existing works therefore limit the analysis to Rayleigh fading channels where the exact pdf of SINR can be derived systematically through taking the Laplace transform at some value [10], [11]. This methodology is however not feasible for other channel distributions of interest [12], and the Rayleigh fading model is not a good fit for practical cases with a direct transmitter-receiver line-of-sight, multiple antennas, etc. [8], [13]. Other principal shortcomings of the direct-pdf approach is the inability to capture the inherent temporal-spatial correlations in the network nodes activities [14]. It should be noted that the need for obtaining the SINR pdf can be precluded using the well-known twostep methodological approach based on Plancherel-Parseval theorem where the coverage probability is first computed, and then the average rate is obtained [1]. While this approach relaxes the Rayleigh fading assumption, it requires evaluating a four-fold integral considering arbitrary fading interfering channels [9].

On the other hand, the average interference power, according to the theory of stable distributions [15], is undefined, and common approximation techniques used to compute the statistics of SINR by taking the ratio of the moments of the denominator and the numerator are not applicable here. Some works thus resort to techniques such as Jensen's inequality [16] and factorial moment expansion [17] to obtain approximate results for the average SINR and its higher-order moments. Employing the former approach provides a lower-bound to the actual result where the tightness depends on the kurtosis of the SINR probability distribution [18]. The latter method is also limited as in most practical cases it requires a large number of terms in the series to provide accurate approximations which comes at the cost of increased computational complexity [19]. The authors in [20] provide a framework for studying the SINR statistics of a typical user in Poisson Networks using factorial moment measures which allows for the derivation of the coverage probability via the inclusion-exclusion principle.

In this paper, we propose a non-direct moment-generatingfunction (MGF) framework for the calculation of the higherorder moments of SINR around an arbitrary PPP-based inter- 
ference field. The employed methodology avoids the need for the computation of the coverage probability and only requires the MGF of the aggregate interference at the reference receiver. At the same time, this model is more computationally-efficient as the number of integrals needed to be evaluated for arbitrary fading channels is reduced to two. Accordingly, closed-form expressions for the statistics of the intended signal and aggregate interference over fading channels involving Nakagami$\mathrm{m}$ and Log-Normal distributions are derived. In particular, we extend the existing results on interference statistics by developing a closed-form expression of the interference MGF considering Nakagami-m fading channels with exclusion region. In addition, explicit expressions for the averages of several different SINR functions of interest are provided. In the special case of path-loss exponent being equal to four, we find closed-form expressions for the pdfs of the aggregate interference reciprocal and signal-to-interference ratio (SIR). In such cases, we prove that useful transmission around a PPPbased deployment of interferers is mathematically equivalent to communicating over a severe one-sided Gaussian fading channel. The proposed methodology is used together with stochastic geometry theory to design and analyze the average SINR and rate performance in heterogeneous cellular networks (HetNets).

The analytical framework can be used to efficiently assess the performance of wireless communication systems in terms of spatial averages. The validity of the mathematical derivations is confirmed through extensive Monte-Carlo simulations for various system setups. Several useful design insights are drawn from our findings. In general, we depict the impact of different parameter settings including channel model, deployment density, noise power, and exclusion zone radius on achievable performance. Furthermore, we assess the performance of a typical user in the HetNet paradigm with interference protection region and show that with network densification there exists a trade-off between the attainable average SINR and rate.

The rest of this paper is organized as follows. Section II describes the PPP-based abstraction model employed throughout this work. In Section III, we present a unified framework for evaluating the exact statistics of SINR. The results concerning the expected value of certain functions of SINR are described in Section IV. The design and analysis of HetNets using stochastic geometry theory is provided in Section V. Theoretical and simulation results together with network design guidelines are given in Section VI. The paper is concluded in Section VII.

Notation: $\mathbb{E}\{$.$\} is the expectation operator; \mathcal{P}_{X}($.$) rep-$ resents the pdf of random variable $\mathrm{X} ; \mathcal{M}_{X}(z)=$ $\mathbb{E}\{\exp (-z X)\}$ denotes the MGF of random variable $X$; $\mathscr{E}_{n}(x)=-\int_{-x}^{+\infty} \frac{\exp (-s)}{s} \mathrm{~d} s$ is the exponential integral function; $\Gamma(x)=\int_{0}^{+\infty} \frac{\exp (-s)}{s^{1-x}} \mathrm{~d} s$ is the Gamma function; $\Gamma(y, x)=\int_{x}^{+\infty} \frac{\exp (-s)}{s^{1-y}} \mathrm{~d} s$ is the (upper) incomplete Gamma function; $\operatorname{erf}(x)=\frac{2}{\sqrt{\pi}} \int_{0}^{x} \exp \left(-s^{2}\right) \mathrm{d} s$ is the Gauss error function; $\operatorname{erfc}(x)=1-\operatorname{erf}(x)$ is the complimentary Gauss error function; $J_{n}(z)=\left(\frac{z}{2}\right)^{n} \sum_{m=0}^{+\infty} \frac{(-1)^{m}}{m ! \Gamma(n+m+1)}\left(\frac{z}{2}\right)^{2 m}$ is the Bessel function of the first kind; $I_{n}=-i^{n} J_{n}(i z)$ is the modified Bessel function of the first kind, where $i=\sqrt{-1}$ is the imaginary unit; $K_{n}(z)=\frac{I_{-n}(z)-I_{n}(z)}{\frac{2}{\pi} \sin (\pi n)}$ represents the modified Bessel function of the second kind; ${ }_{2} F_{1}(a, b ; c ; z)=\sum_{x=0}^{+\infty} \frac{(a)_{x}(b)_{x}}{(c)_{x} x !} z^{x}$ is the Gauss hypergeometric function, where $(n)_{x}=n(n+1) \ldots(n+x-$ $1) ;{ }_{2} \tilde{F}_{1}(a, b ; c ; z)=\frac{{ }_{2} F_{1}(a, b ; c ; z)}{\Gamma(c)}$ is the Regularized Gauss hypergeometric function; ${ }_{1} F_{1}(a ; b ; z)=\sum_{x=0}^{+\infty} \frac{(a)_{x}}{(b)_{x}} \frac{z^{x}}{x !}$ is the confluent hypergeometric function of the first kind; ${ }_{1} \tilde{F}_{1}(a ; b ; z)=\frac{1 F_{1}(a, b ; z)}{\Gamma(b)}$ is the Regularized confluent hypergeometric function of the first kind; and $U(a ; b ; z)=$ $\pi \csc (\pi b)\left[\frac{1 \tilde{1}_{1}(a ; b ; z)}{\Gamma(a-b+1)}-\frac{{ }_{1} \tilde{F}_{1}(a-b+1 ; b-b ; z)}{z^{b-1} \Gamma(a)}\right]$ is the confluent hypergeometric function of the second kind, where $\csc (\pi b)=$ $\frac{1}{\pi b} \Gamma(1-b) \Gamma(1+b)$.

\section{SySTEM MOdeL}

We consider a receiver experiencing interference from $T$ different classes of sources distributed according to independent stationary PPPs $\Phi_{t}$ with densities $\lambda_{t}$, where $t \in \mathcal{T}=$ $\{1,2, \ldots T\}$. The PPP-based abstraction model was recently shown to be an accurate representation for the locations of dense, irregular, and overlapping nodes in emerging cellular networks [21]. Adopting PPPs resembles randomized medium access control (MAC) layer protocols where interferers access the channel in an ALOHA-like manner. Note that by utilizing the Mapping theorem in a similar approach to that in [22], the PPPs can be transferred into an equivalent PPP with unity fading, then all the different random aspects are captured in the intensity function.

Throughout the paper, we consider the case where all nodes are equipped with a single-antenna. Multi-antenna communication can however be incorporated in the proposed framework by applying similar methodological approaches to those proposed in [23] and [24]. In addition, here, a noncooperative transmission protocol is considered. The work in [25] provides a tractable stochastic geometry-based model considering several different cooperative mechanisms.

Let $g_{0}, \psi_{0}$, and $r_{0}$ respectively denote the fading power gain, shadowing power gain, and distance of the reference receivertransmitter pair. Moreover, for the $k$-th tier- $t$ interferer, $g_{t, k}$, $\psi_{t, k}$, and $r_{t, k}, t \in \mathcal{T}, k \in \Phi_{t}$, are used to represent the fading power gain, shadowing power gain, and distance with respect to the victim receiver, respectively. The corresponding SINR of the reference receiver can hence be written as

$$
\gamma=\frac{X_{0}}{I_{a g g}+\eta}=\frac{g_{0} \psi_{0} r_{0}^{-\beta}}{\sum_{t \in \mathcal{T}} \sum_{k \in \Phi_{t}} g_{t, k} \psi_{t, k} r_{t, k}^{-\beta}+\eta}
$$

where $\eta$ is the variance of the additive white noise and $\beta$ $(>2)$ is the path-loss exponent. It is important to note that the distance-dependent path-loss function in (1) is unbounded as a result of its singularity at the origin. However, employing more practical path-loss models, e.g., the modified power-law function $\min \left(1, r^{-\beta}\right)$, complicates the analysis [26]. Hence, for the sake of analytical tractability, we utilize the unbounded path-loss model in this paper. 


\section{SINR STATISTICS IN A POISSON FIELD OF INTERFERERS}

By using the MGF-based approach from our previous work in [27, eq. (5)], the statistical moments of SINR can be calculated using the expression in the following theorem.

Theorem 1. Let $\mathbb{E}\left\{X_{0}^{n}\right\}$ and $\mathcal{M}_{I_{\text {agg }}}(z)$ respectively denote the $n$-th moment of intended signal power and MGF of aggregate interference, then, the $n$-th positive moment $(n>0)$ of the SINR of a reference receiver can be expressed as

$$
\mathbb{E}\left\{\gamma^{n}\right\}=\mathbb{E}\left\{X_{0}^{n}\right\} \int_{0}^{+\infty} \frac{z^{n-1}}{\Gamma(n)} \mathcal{M}_{I_{\text {agg }}}(z) \exp (-z \eta) \mathrm{d} z .
$$

Proof: See Appendix A.

We calculate the $n$-th moment of the useful signal power for various different channel distributions of interest. Specifically, we consider Gamma faded power gain with Nakagami-m parameter $m\left(\geq \frac{1}{2}\right)$ and mean squared value $\Omega(>0)$ and LogNormal shadowed power gain with mean $\mu(\in \mathbb{R})$ and standard deviation $\sigma(>0)$. Unless otherwise stated, we consider a fixed unit-distance between the reference transmitter-receiver pair (i.e., $r_{0}=1$ ). The analysis considering arbitrary values of $r_{0}$ with exclusion region using stochastic geometry theory is provided in Section V. The results are presented in the following lemma.

Lemma 1. The n-th positive moment of the intended signal power considering independent Nakagami-m fading and LogNormal shadowing channel gains is given by

$$
\mathbb{E}\left\{X_{0}^{n}\right\}=\frac{\Gamma(n+m)}{\Gamma(m)}\left(\frac{\Omega}{m}\right)^{n} \exp \left(\frac{1}{2}(n \sigma)^{2}+\mu n\right) .
$$

Without the effect of shadowing, we can obtain in the case of Nakagami-m fading

$$
\mathbb{E}\left\{X_{0}^{n}\right\}=\frac{\Gamma(n+m)}{\Gamma(m)}\left(\frac{\Omega}{m}\right)^{n} .
$$

For the special case of Rayleigh fading

$$
\mathbb{E}\left\{X_{0}^{n}\right\}=\Gamma(1+n) \Omega^{n} .
$$

With Log-Normal shadowing only, we have

$$
\mathbb{E}\left\{X_{0}^{n}\right\}=\exp \left(\frac{1}{2}(n \sigma)^{2}+\mu n\right) .
$$

Proof: See Appendix B.

Next, we provide a closed-form expression for $\mathcal{M}_{I_{a g g}}(z)$ using a new systematic approach different from the proof for homogeneous sources in our previous work in [28]. We consider uncorrelated heterogeneous interfering links using Gamma faded power gains with Nakagami-m parameters $m_{t}$ $\left(\geq \frac{1}{2}\right)$ and mean squared values $\Omega_{t}(>0)$ and Log-Normal shadowed power gains with means $\mu_{t}(\in \mathbb{R})$ and standard deviations $\sigma_{t}(>0)$, where $t \in \mathcal{T}$. However, it is important to note that in several communication techniques and protocols, the fluctuations of the interference power is correlated across time and/or space. The reader is referred to [29] and [30] concerning the impact of interference dynamics on system design and performance.
Lemma 2. The MGF of the aggregate interference over independent Nakagami-m fading and Log-Normal shadowing interfering links is given by

$$
\mathcal{M}_{I_{\text {agg }}}(z)=\exp \left(-z^{\frac{2}{\beta}} \mathcal{A}\right)
$$

where

$$
\mathcal{A}=\pi \Gamma\left(1-\frac{2}{\beta}\right) \sum_{t \in \mathcal{T}} \lambda_{t} \Omega_{t}^{\frac{2}{\beta}} \frac{\Gamma\left(m_{t}+\frac{2}{\beta}\right)}{m_{t}^{\frac{2}{\beta}} \Gamma\left(m_{t}\right)} \exp \left(\frac{2}{\beta}\left(\mu_{t}+\frac{\sigma_{t}^{2}}{\beta}\right)\right) .
$$

Without shadowing, we respectively obtain for Nakagami-m and Rayleigh fading interfering links

$$
\mathcal{A}=\pi \Gamma\left(1-\frac{2}{\beta}\right) \sum_{t \in \mathcal{T}} \lambda_{t} \Omega_{t}^{\frac{2}{\beta}} \frac{\Gamma\left(m_{t}+\frac{2}{\beta}\right)}{m_{t}^{\frac{2}{\beta}} \Gamma\left(m_{t}\right)}
$$

and

$$
\mathcal{A}=\pi \Gamma\left(1-\frac{2}{\beta}\right) \sum_{t \in \mathcal{T}} \lambda_{t} \Omega_{t}^{\frac{2}{\beta}} \Gamma\left(1+\frac{2}{\beta}\right) .
$$

Considering Log-Normal shadowing only, $\mathcal{A}$ is expressed as

$$
\mathcal{A}=\pi \Gamma\left(1-\frac{2}{\beta}\right) \sum_{t \in \mathcal{T}} \lambda_{t} \exp \left(\frac{2}{\beta}\left(\mu_{t}+\frac{\sigma_{t}^{2}}{\beta}\right)\right) .
$$

Proof: See Appendix C.

It is important to note that most practical multiple access techniques (except ALOHA) guarantee interference protection by imposing a restricted area around the receiver where no transmission can take place. In the following lemma, we provide a closed-form expression of the aggregate interference statistics over Nakagami-m fading channels considering an exclusion zone of radius $r_{e}$ around the reference receiver.

Lemma 3. The MGF of the aggregate interference over Nakagami-m fading channels under the impact of exclusion zone can be derived as

$$
\begin{aligned}
& \mathcal{M}_{I_{\text {agg }}}(z)=\exp \left(-\pi \sum_{t \in \mathcal{T}} \lambda_{t}\left[r_{e}^{2}\left(\left(\frac{m_{t}}{z \Omega_{t} r_{e}^{-\beta}+m_{t}}\right)^{m_{t}}-1\right)\right.\right. \\
& +\Gamma\left(m_{t}+\frac{2}{\beta}\right)\left(\frac{\Gamma\left(1-\frac{2}{\beta}\right)}{\Gamma\left(m_{t}\right)}\left(\frac{z \Omega_{t}}{m_{t}}\right)^{\frac{2}{\beta}}-m_{t}\left(\frac{m_{t} r_{e}^{\frac{2}{m_{t}}+\beta}}{z \Omega_{t}}\right)^{m_{t}}\right. \\
& \left.\left.\left.\times{ }_{2} \tilde{F}_{1}\left(m_{t}+1, m_{t}+\frac{2}{\beta} ; m_{t}+\frac{2}{\beta}+1 ;-\frac{m_{t} r_{e}^{\beta}}{z \Omega_{t}}\right)\right)\right]\right) .
\end{aligned}
$$

In the case of Rayleigh fading interfering channels

$$
\begin{aligned}
& \mathcal{M}_{I_{\text {agg }}}(z)=\exp \left(-\pi \sum_{t \in \mathcal{T}} \lambda_{t}\left[\left(z \Omega_{t}\right)^{\frac{2}{\beta}} \Gamma\left(1-\frac{2}{\beta}\right) \Gamma\left(1+\frac{2}{\beta}\right)\right.\right. \\
& \left.\left.-r_{e}^{2}\left(1-\frac{2 r_{e}^{\beta}}{z \Omega_{t}(\beta+2)}{ }_{2} F_{1}\left(1,1+\frac{2}{\beta} ; 2+\frac{2}{\beta} ;-\frac{r_{e}^{\beta}}{z \Omega_{t}}\right)\right)\right]\right) .
\end{aligned}
$$


For the special case of path-loss exponent being equal to four, the above can be simplified to

$$
\mathcal{M}_{I_{\text {agg }}}(z)=\exp \left(-\pi \sum_{t \in \mathcal{T}} \lambda_{t}\left[\sqrt{z \Omega_{t}} \arctan \left(\frac{\sqrt{z \Omega_{t}}}{r_{e}^{2}}\right)\right]\right) .
$$

\section{Proof: See Appendix D.}

Notice that the interference follows a stable distribution with $\beta>2$, implying that all moments greater than $\frac{2}{\beta}$ (such as the average interference) are infinite. The expression in (2) nevertheless holds considering the integrand which involves the MGF of the interference converges under the unbounded path-loss model with $\beta>2$.

Utilizing the closed-form expressions of the statistics of the useful signal and aggregate interference in the proposed analytical framework facilitates efficient computation of the exact higher-order moments of SINR under arbitrary signal-tonoise ratios (SNRs). Without loss of generality, we henceforth consider intended fading channel distributions of unit mean squared value with $\Omega=1$ and $\mu=-\frac{\sigma^{2}}{2}$. Similarly, normalized mean squared values for interfering links are hereafter assumed with $\Omega_{t}=1$ and $\mu_{t}=-\frac{\sigma_{t}^{2}}{2}$, where $t \in \mathcal{T}$.

In the remaining parts of this section, we focus on various special cases of interest in which it is possible to derive closed-form results for the SINR statistics. The analysis is only valid for cases with no protection region as a consequence of the extra terms in the interference MGF in Lemma 3 which can no longer be expressed in the analytically-tractable form $\mathbb{E}\left\{\exp \left(-z^{2 / \beta} \mathcal{A}\right)\right\}$.

The wireless environment in certain practical scenarios such as dense urban areas is interference-limited. The impact of background noise in such cases is considered negligible. Deriving closed-form results for the network performance is particularly attractive in this case due to the rapid deployment of antennas and base stations aimed at meeting the overwhelming traffic forecasts. For interference-limited environment (when $\eta \rightarrow 0$ ), the $n$-th positive moment of SINR admits a closedform expression as presented in Lemma 4.

Lemma 4. The $n$-th positive moment of SINR in interferencelimited scenarios is given by

$$
\mathbb{E}\left\{\gamma^{n}\right\}=\frac{\Gamma\left(\frac{n \beta}{2}+1\right)}{\Gamma(n)} \mathbb{E}\left\{X_{0}^{n}\right\} \mathcal{A}^{\frac{-n \beta}{2}} .
$$

The respective average SINR is therefore

$$
\mathbb{E}\{\gamma\}=\Gamma\left(\frac{\beta}{2}+1\right) \mathbb{E}\left\{X_{0}\right\} \mathcal{A}^{\frac{-\beta}{2}} .
$$

Proof: See Appendix E.

On the other hand, when considering the impact of non-zero additive noise power, we derive a closed-form expression for the statistics of SINR in the special case of $\beta=4$ as in the following lemma.

Lemma 5. The $n$-th positive moment of SINR with non-zero noise power and $\beta=4$ is given by

$$
\mathbb{E}\left\{\gamma^{n}\right\}=\frac{1}{\eta^{n}} \mathbb{E}\left\{X_{0}^{n}\right\}\left({ }_{1} F_{1}\left(n ; \frac{1}{2} ; \frac{1}{4 \eta} \mathcal{A}^{2}\right)\right.
$$

$$
\left.-\frac{\Gamma\left(n+\frac{1}{2}\right)}{\sqrt{\eta} \Gamma(n)} \mathcal{A}_{1} F_{1}\left(n+\frac{1}{2} ; \frac{3}{2} ; \frac{1}{4 \eta} \mathcal{A}^{2}\right)\right) .
$$

The above can be alternatively expressed as

$$
\mathbb{E}\left\{\gamma^{n}\right\}=\frac{2^{1-2 n} \Gamma(2 n)}{\Gamma(n) \eta^{n}} \mathbb{E}\left\{X_{0}^{n}\right\} U\left(n, \frac{1}{2}, \frac{1}{4 \eta} \mathcal{A}^{2}\right) .
$$

The first moment of SINR for arbitrary non-zero noise variance can be expressed as

$$
\mathbb{E}\{\gamma\}=\frac{1}{\eta}\left(1-\frac{1}{2 \sqrt{\eta}} \sqrt{\pi} \mathcal{A} \exp \left(\frac{1}{4 \eta} \mathcal{A}^{2}\right) \operatorname{erfc}\left(\frac{1}{2 \sqrt{\eta}} \mathcal{A}\right)\right) .
$$

\section{Proof: See Appendix F.}

\section{Some ApPliCATIONS OF SINR FUnCtions}

As an important application of SINR statistics, here, we derive explicit expressions for the averages of certain functions which appear commonly in the performance evaluation of wireless communication systems. Note that the analytical tractability of the techniques employed to derive the closedform expressions in this section quickly diminishes under interference exclusion region. Hence, unless otherwise stated, the results are obtained assuming $r_{e}=0$.

\section{A. Average of the Complimentary Error Function: $\mathbb{E}\{\operatorname{erfc}(\sqrt{\gamma})\}$}

To develop an expression for the average of the complimentary error function, we can write

$$
\begin{aligned}
\operatorname{erfc}(\sqrt{\gamma}) & =\frac{2}{\pi} \int_{0}^{\frac{\pi}{2}} \exp \left(-\gamma \csc ^{2}(\theta)\right) \mathrm{d} \theta \\
& =1-\frac{2}{\sqrt{\pi}} \sum_{n=0}^{+\infty} \frac{(-1)^{n}}{n !(2 n+1)} \gamma^{n+\frac{1}{2}} .
\end{aligned}
$$

It is then possible to derive a generalized expression of $\mathbb{E}\{\operatorname{erfc}(\sqrt{\gamma})\}$ considering the intended signal power follows a Gamma distribution and interference is a non-negative arbitrary random variable as in [28, eq. (10)]. In this paper, we show that for interference-limited environment with the special case of $\beta=4$, a strikingly simple expression for $\mathbb{E}\left\{\operatorname{erfc}(\sqrt{\gamma}) \mid X_{0}\right\}$ can be developed. Furthermore, we derive a closed-form expression for $\mathbb{E}\{\operatorname{erfc}(\sqrt{\gamma})\}$ in the case of Nakagami-m fading channels. The results are presented in the following lemma.

Lemma 6. The average of the complimentary error function of SINR conditioned on the useful signal power in interferencelimited scenarios with $\beta=4$ is given by

$$
\mathbb{E}\left\{\operatorname{erfc}(\sqrt{\gamma}) \mid X_{0}\right\}=\frac{2}{\pi} \arctan \left(\frac{\mathcal{A}}{2 \sqrt{X_{0}}}\right) .
$$

Given the intended signal experiences Nakagami-m fading, we can obtain

$$
\begin{aligned}
& \mathbb{E}\{\operatorname{erfc}(\sqrt{\gamma})\}=\frac{1}{\Gamma(m)}\left(\frac{\Gamma(m)-\Gamma\left(m,-\frac{1}{4}\left(\mathcal{A}^{2} m\right)\right)}{(-1)^{m} \cos (\pi m)}\right. \\
& \left.+\frac{\mathcal{A} \sqrt{m} \Gamma\left(m-\frac{1}{2}\right)}{\pi}{ }_{2} F_{2}\left(\frac{1}{2}, 1 ; \frac{3}{2}, \frac{3}{2}-m ; \frac{\mathcal{A}^{2} m}{4}\right)\right) .
\end{aligned}
$$


Considering the special case of Rayleigh faded intended link, we have

$$
\mathbb{E}\{\operatorname{erfc}(\sqrt{\gamma})\}=1-\exp \left(\frac{\mathcal{A}^{2}}{4}\right) \operatorname{erfc}\left(\frac{\mathcal{A}}{2}\right) .
$$

Proof: See Appendix G.

It should be noted that a closed-form expression for $\mathbb{E}\{\operatorname{erfc}(\sqrt{\gamma})\}$ does not exist for channel distributions involving Log-Normal shadowing. However the conditional expression developed in (21) facilitates efficient numerical computation of average complimentary error function since the pdfs of common fading distributions are readily available in the literature.

\section{B. Average of the Exponential Function: $\mathbb{E}\{\exp (-\gamma)\}$}

Consider the following Taylor series expansion

$$
\exp (-\gamma)=1+\sum_{n=1}^{+\infty} \frac{(-1)^{n}}{n !} \gamma^{n}
$$

$\mathbb{E}\{\exp (-\gamma)\}$ can be easily numerically computed for Gammadistributed signal power and arbitary interference using the generalized expression in [28, eq. (11)]. For interferencelimited case with $\beta=4$, a closed-form expression for $\mathbb{E}\{\exp (-\gamma)\}$ is developed as shown below.

Lemma 7. The average of the exponential function of SINR conditioned on the useful signal power in interference-limited scenarios with $\beta=4$ is given by

$$
\mathbb{E}\left\{\exp (-\gamma) \mid X_{0}\right\}=\frac{\mathcal{A}}{\sqrt{\mathcal{A}^{2}+4 X_{0}}} .
$$

When the useful signal experiences Nakagami-m fading, we can obtain

$$
\begin{aligned}
\mathbb{E}\{\exp (-\gamma)\} & =\frac{\sqrt{\pi} m^{m} \mathcal{A}^{2 m}}{4^{m} \cos (\pi m)}{ }_{1} \tilde{F}_{1}\left(m ; m+\frac{1}{2} ; \frac{\mathcal{A}^{2} m}{4}\right) \\
& -\frac{\pi \mathcal{A} \sqrt{m}}{2 \Gamma(m) \cos (\pi m)}{ }_{1} \tilde{F}_{1}\left(\frac{1}{2} ; \frac{3}{2}-m ; \frac{\mathcal{A}^{2} m}{4}\right) .
\end{aligned}
$$

For the special case of Rayleigh fading channel model, we obtain

$$
\mathbb{E}\{\exp (-\gamma)\}=\frac{1}{2} \sqrt{\pi} \mathcal{A} \exp \left(\frac{\mathcal{A}^{2}}{4}\right) \operatorname{erfc}\left(\frac{\mathcal{A}}{2}\right) .
$$

Proof: See Appendix H.

The MGF of a random variable (if it exists) is related to the Laplacian of the density function. However, it is generally very difficult to invert the MGF for the purpose of recovering the pdf. In the following lemma, we show that it is possible to develop closed-form expressions for the density functions of aggregate interference reciprocal and SIR when $\beta=4$.

Lemma 8. The pdf of PPP-based aggregate interference reciprocal with $\eta=0$ and $\beta=4$ is

$$
\mathcal{P}_{\frac{1}{I_{\text {agg }}}}(x)=\frac{\mathcal{A}}{2 \sqrt{\pi x}} \exp \left(-\frac{\mathcal{A}^{2} x}{4}\right) .
$$

With Nakagami-m faded useful link, the respective pdf of SIR has an analytical form given by

$$
\mathcal{P}_{\gamma}(x)=\frac{2^{\frac{1}{2}-m} m^{\frac{m}{2}+\frac{1}{4}}}{\sqrt{\pi} \Gamma(m)} \mathcal{A}^{m+\frac{1}{2}} x^{\frac{m}{2}-\frac{3}{4}} K_{m-\frac{1}{2}}(\mathcal{A} \sqrt{m x}) .
$$

With Rayleigh faded intended channel environment, the above reduces to

$$
\mathcal{P}_{\gamma}(x)=\frac{\mathcal{A}}{2 \sqrt{x}} \exp (-\mathcal{A} \sqrt{x}) .
$$

Proof: See Appendix I.

It is important to remind that the closed-form expressions presented in the above lemma can be viewed as limited in particular considering that the unbounded path-loss model highly overestimates the interference power. Nevertheless, in this special case, the inverse of the total power from the PPPbased abstraction model of interfering nodes can be modeled via a single random variable as discussed in the following corollary.

Corollary 1 (Equivalence Interference Model). The pdf of the reciprocal of total PPP-based interference power in an interference-limited scenario with the special case of pathloss exponent being equal to four is equivalent to a Gamma distribution with Nakagami-m fading parameter $\frac{1}{2}$ and mean $\frac{2}{\mathcal{A}^{2}}$. We can therefore infer that in such cases the effect of interference on useful signal is mathematically equivalent to transmitting over a severe one-sided Gaussian fading channel.

\section{Average of the Logarithm Function: $\mathbb{E}\{\log (1+\gamma)\}$}

The ergodic capacity over fading interference channels considering mutually independent random variables can be expressed in terms of a Laguerre orthogonal polynomial as [31]

$$
\begin{aligned}
\mathbb{E}\{\log (1+\gamma)\} & =\sum_{e=1}^{E} \frac{w_{e}}{a_{e}} \exp \left(-a_{e}^{\frac{2}{\beta}} \mathcal{A}\right)\left[1-\mathcal{M}_{X_{0}}\left(a_{e}\right)\right] \\
& \times \exp (-z \eta)+R_{E}
\end{aligned}
$$

where $w_{e}$ and $a_{e}$ are the weights and abscissae of the $E$ th order Hermite polynomial, $\mathcal{M}_{X_{0}}($.$) denotes the MGF of$ the intended signal, and $R_{E}$ is a remainder, respectively. It is widely known that integral transformations involving LogNormal distribution do not exist in closed-form. The following tractable MGF expression based on Gaussian quadrature numerical method is however shown to be a reasonable approximation for composite Nakagami-m/Log-Normal fading. Considering normalized mean squared fading values, we have [32]

$$
\begin{aligned}
\mathcal{M}_{X_{0}}\left(a_{e}\right) & =\mathbb{E}\left\{\exp \left(-a_{e} g_{0} \psi_{0}\right)\right\} \\
& \approx \frac{1}{\sqrt{\pi}} \sum_{\theta=1}^{\Theta} w_{\theta}\left(1-\frac{\left(\frac{\sigma}{2}-\sqrt{2} a_{\theta}\right) \sigma}{m} a_{e}\right)^{-m}
\end{aligned}
$$

where $w_{\theta}$ and $a_{\theta}$ are the $\Theta$-order Hermite polynomial weights and abscissae, respectively. It is worth mentioning that the SINR moments can be utilized to determine the higher-order amount of fading in order to obtain asymptotic results on the statistics of capacity, as explained in [33]. 


\section{Stochastic Transmitter-Receiver Geometry}

In this section, we relax the unit-distance assumption between the reference transmitter-receiver pair using stochastic geometry theory. This approach has applications in HetNets. Without loss of generality, by employing Mecke's theorem [34], the analysis is carried out for an arbitrary user assumed to be located at the center. Throughout this section, heterogeneous deployed nodes are considered to be base stations (BSs) with different spatial densities $\lambda_{t}$ and transmit powers $p_{t}$, where $t \in \mathcal{T}$. A co-channel HetNet deployment with universal frequency reuse allowing all cells to utilize the entire system spectrum is taken into account.

We consider a cellular association strategy where the reference user connects to the closest BS of a certain tier which provides the strongest received signal power mathematically formulated as

$$
t^{*}=\underset{t \in \mathcal{T}}{\arg \max }\left(p_{t} d_{t}^{-\beta}\right)
$$

where $d_{t} \equiv r_{t, k_{t}}$ denotes the distance of the closest tier- $t$ BS $k_{t}$ to the reference user. The effect of exclusion zone is considered where the distance of the transmitter-receiver pair must be at least $r_{e}(>0)$. Hence, with a minor modification of [35, Appendix C] to include the effect of exclusion zone, the pdf of the statistical transmitter-receiver distance $\left(r_{0}>r_{e}\right)$ can be written as

$$
\begin{aligned}
\mathcal{P}_{d_{t^{*}}}\left(r_{0}\right) & =\exp \left(-\pi\left(r_{0}-r_{e}\right)^{2} \sum_{t \in \mathcal{T}} \lambda_{t}\left(\frac{p_{t}}{p_{t^{*}}}\right)^{\frac{2}{\beta}}\right) \\
& \times \frac{2 \pi\left(r_{0}-r_{e}\right) \lambda_{t^{*}}}{\mathscr{P}_{t^{*}}}
\end{aligned}
$$

where $\mathscr{P}_{t^{*}}$ denotes the probability of the event that the reference user is associated with the closest tier- $t^{*}$ BS expressed by [35]

$$
\mathscr{P}_{t^{*}}=\frac{\lambda_{t^{*}}}{\sum_{t \in \mathcal{T}} \lambda_{t}\left(\frac{p_{t}}{p_{t^{*}}}\right)^{\frac{2}{\beta}}} .
$$

The respective positive $n$-th order moment of SINR of a typical user in the downlink HetNet under consideration can be expressed as

$$
\mathbb{E}\left\{\gamma^{n}\right\}=\sum_{t^{*} \in \mathcal{T}} \mathbb{E}\left\{\gamma_{t^{*}}^{n}\right\} \mathscr{P}_{t^{*}}
$$

where $\gamma_{t^{*}}$ is the instantaneous SINR the closest tier- $t^{*}$ BS provides

$$
\gamma_{t^{*}}=\frac{X_{0}}{I_{a g g}+\eta}=\frac{p_{t^{*}} g_{t^{*}, k_{t^{*}}} r_{t^{*}, k_{t^{*}}}^{-\beta}}{\sum_{t \in \mathcal{T}} \sum_{k \in \Phi_{t} /\left\{k_{t^{*}}\right\}} p_{t} g_{t, k} r_{t, k}^{-\beta}+\eta} .
$$

Note that shadowing effects can be easily incorporated in the proposed framework by adjusting the transmit powers of different tiers according to the displacement theorem [36], [37].

Utilizing the result in Theorem 1, the higher-order statistics of the SINR of an arbitrary user associated with the closest tier- $t^{*} \mathrm{BS}$ in the HetNet under consideration can be expressed as [38]

$$
\begin{aligned}
\mathbb{E}\left\{\gamma_{t^{*}}^{n}\right\} & =\int_{r_{e}}^{+\infty} \mathbb{E}\left\{X_{0}^{n} \mid r_{0}\right\} \int_{0}^{+\infty} \mathcal{M}_{I_{a g g} \mid r_{0}}(z) \exp (-z \eta) \\
& \times \mathcal{P}_{d_{t^{*}}}\left(r_{0}\right) \mathrm{d} z \mathrm{~d} r_{0}
\end{aligned}
$$

The positive moments of the intended signal power with arbitrary transmitter-receiver distance do not admit closedform expressions in terms of elementary functions. $\mathbb{E}\left\{X_{0}^{n}\right\}$ can however be easily computed for different channel models through numerical integration of the developed expressions in Lemma 2 with respect to the statistical distance $r_{0}$. On the other hand, a main contribution of this work, is in deriving a closed-form expression for $\mathcal{M}_{I_{a g g} \mid r_{0}}(z)$ over Nakagami-m fading interfering links as presented in the following lemma.

Lemma 9. The aggregate network interference MGF over Nakagami-m fading channels conditioned on the distance between reference transmitter-receiver pair being $r_{0}$ is given by (39). In the case of Rayleigh fading interfering channels we obtain (40). For the special case of path-loss exponent being equal to four, (41) is derived.

Proof: The result can be obtained using a similar approach to that in Appendix $D$ with $r_{0}\left(p_{t} / p_{t^{*}}\right)^{\frac{1}{\beta}}$ being the lower-bound on the distance of the closest tier-t interferer.

It is useful to note that the closed-form expression of the aggregate interference developed in Lemma 9 can be used to compute the exact average rate (nat/s/Hz) in the HetNet paradigm using the following computationally-efficient MGFbased methodology [38, Lemma 1]

$$
\mathbb{E}\{\log (1+\gamma)\}=\sum_{t^{*} \in \mathcal{T}} \mathbb{E}\left\{\log \left(1+\gamma_{t^{*}}\right)\right\} \mathscr{P}_{t^{*}}
$$

By denoting the MGF of the intended signal conditioned on the distance of the arbitrary transmitter-receiver being $r_{0}$ with $\mathcal{M}_{X_{0} \mid r_{0}}(z)$, we have

$$
\begin{aligned}
\mathbb{E}\left\{\log \left(1+\gamma_{t^{*}}\right)\right\} & =\int_{r_{e}}^{+\infty} \int_{0}^{+\infty}\left[\left(1-\mathcal{M}_{X_{0} \mid r_{0}}(z)\right)\right. \\
& \left.\times \mathcal{M}_{I_{a g g} \mid r_{0}}(z)\right] \frac{\exp (-z \eta)}{z} \mathcal{P}_{d_{t^{*}}}\left(r_{0}\right) \mathrm{d} z \mathrm{~d} r_{0}
\end{aligned}
$$

Closed-form expressions for the MGF of different fading distributions are readily available in the literature, hence, $\mathcal{M}_{X_{0} \mid r_{0}}(z)$ can be easily computed, e.g., in the case of normalized Nakagami-m faded intended link we have

$$
M_{X_{0} \mid r_{0}}(z)=\left(1+\frac{z p_{t^{*}}}{m_{t^{*}} r_{0}^{\beta}}\right)^{-m_{t^{*}}}
$$

In summary, the proposed framework relaxes the longstanding assumption of Rayleigh distribution for interference characterization in the existing literature and allows for computation of the HetNet exact average SINR and rate for general cellular network setups via double integrals - as opposed to the manifold numerical integrals the classical direct-pdf approach requires. 


$$
\begin{aligned}
\mathcal{M}_{I_{\text {agg }} \mid r_{0}}(z) & =\exp \left(-\pi \sum_{t \in \mathcal{T}} \lambda_{t}\left[r_{0}^{2}\left(\frac{p_{t}}{p_{t^{*}}}\right)^{\frac{2}{\beta}}\left(\left(\frac{m_{t}}{z p_{t^{*}} r_{0}^{-\beta}+m_{t}}\right)^{m_{t}}-1\right)+p_{t}^{\frac{2}{\beta}} \Gamma\left(m_{t}+\frac{2}{\beta}\right)\left(\frac{\Gamma\left(1-\frac{2}{\beta}\right)}{\Gamma\left(m_{t}\right)}\left(\frac{z}{m_{t}}\right)^{\frac{2}{\beta}}\right.\right.\right. \\
& \left.\left.\left.-\frac{m_{t}}{p_{t^{*}}^{\frac{2}{\beta}}}\left(\frac{m_{t} p_{t^{*}} r_{0}^{\frac{2}{m_{t}}+\beta}}{z}\right)^{m_{t}}{ }_{2} \tilde{F}_{1}\left(m_{t}+1, m_{t}+\frac{2}{\beta} ; m_{t}+\frac{2}{\beta}+1 ;-\frac{m_{t} r_{0}^{\beta}}{z p_{t^{*}}}\right)\right)\right]\right)
\end{aligned}
$$

$$
\begin{aligned}
\mathcal{M}_{I_{\text {agg }} \mid r_{0}}(z) & =\exp \left(-\pi \sum_{t \in \mathcal{T}} \lambda_{t}\left[\left(z p_{t}\right)^{\frac{2}{\beta}} \Gamma\left(1-\frac{2}{\beta}\right) \Gamma\left(1+\frac{2}{\beta}\right)\right.\right. \\
& \left.\left.-r_{0}^{2}\left(\frac{p_{t}}{p_{t^{*}}}\right)^{\frac{2}{\beta}}\left(1-\frac{2 r_{0}^{\beta}}{z p_{t^{*}}(\beta+2)}{ }_{2} F_{1}\left(1,1+\frac{2}{\beta} ; 2+\frac{2}{\beta} ;-\frac{r_{0}^{\beta}}{z p_{t^{*}}}\right)\right)\right]\right)
\end{aligned}
$$

$$
\mathcal{M}_{I_{a g g} \mid r_{0}}(z)=\exp \left(-\pi \sum_{t \in \mathcal{T}} \lambda_{t}\left[\sqrt{z p_{t}} \arctan \left(\frac{\sqrt{z p_{t^{*}}}}{r_{0}^{2}}\right)\right]\right)
$$

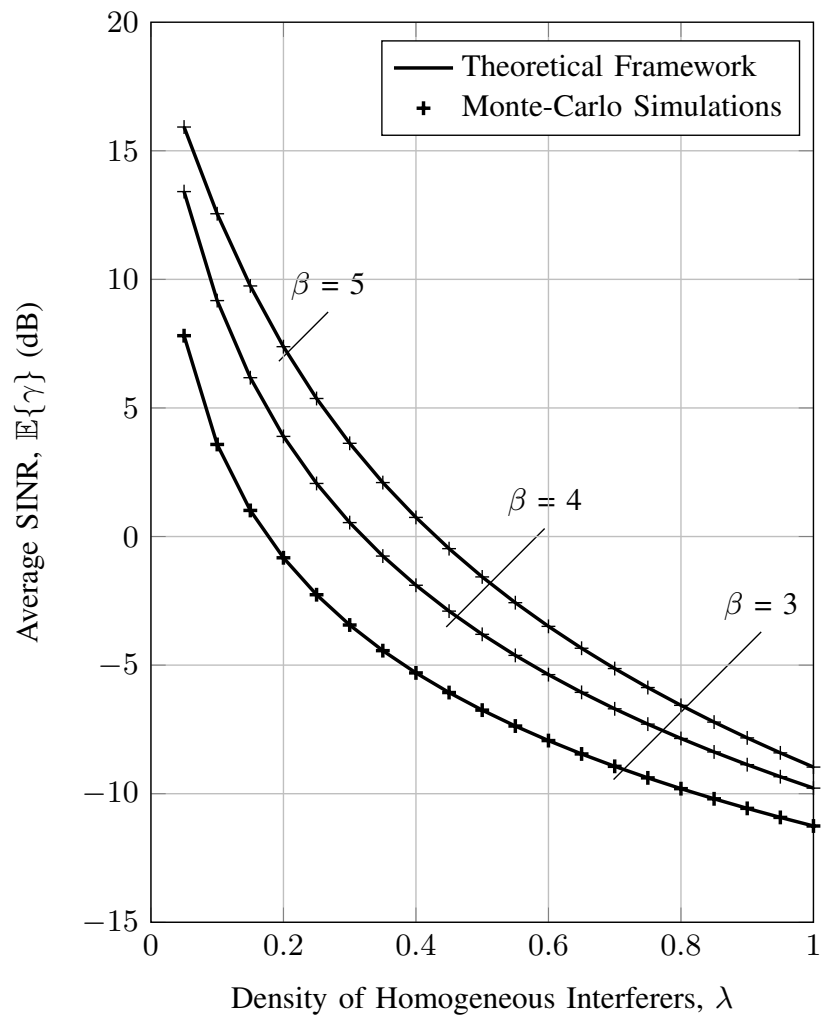

Fig. 1: Effect of homogeneous interferers density on the average SINR of an arbitrary transceiver. System parameters are: $\mathbb{E}\left\{X_{0}\right\}=1$, $\frac{1}{\eta}=20 \mathrm{~dB}, T=1, m_{1}=1, \sigma_{1}=1, r_{e}=0$.

\section{Vi. Performance Evaluation}

In order to verify the accuracy and reliability of the proposed analytical framework, we present several examples to compare the theoretical results with Monte-Carlo simulations.

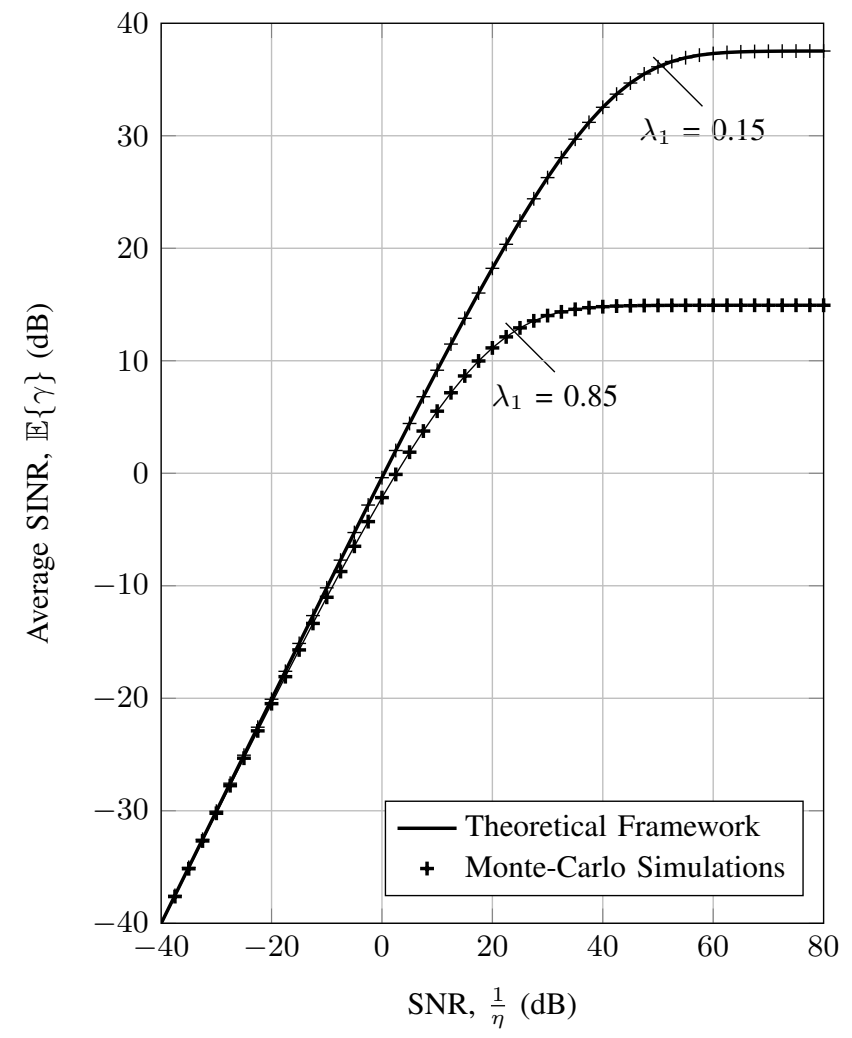

Fig. 2: Impact of different noise variance values on the average SINR of an arbitrary transceiver. System parameters are: $\mathbb{E}\left\{X_{0}\right\}=1, \beta=$ $6, T=1, m_{1}=2, \sigma_{1}=4, r_{e}=0$.

We further aim to quantify the dependence and trade-offs of different parameter settings on achievable performance which helps unveil interesting trends and design guidelines. 


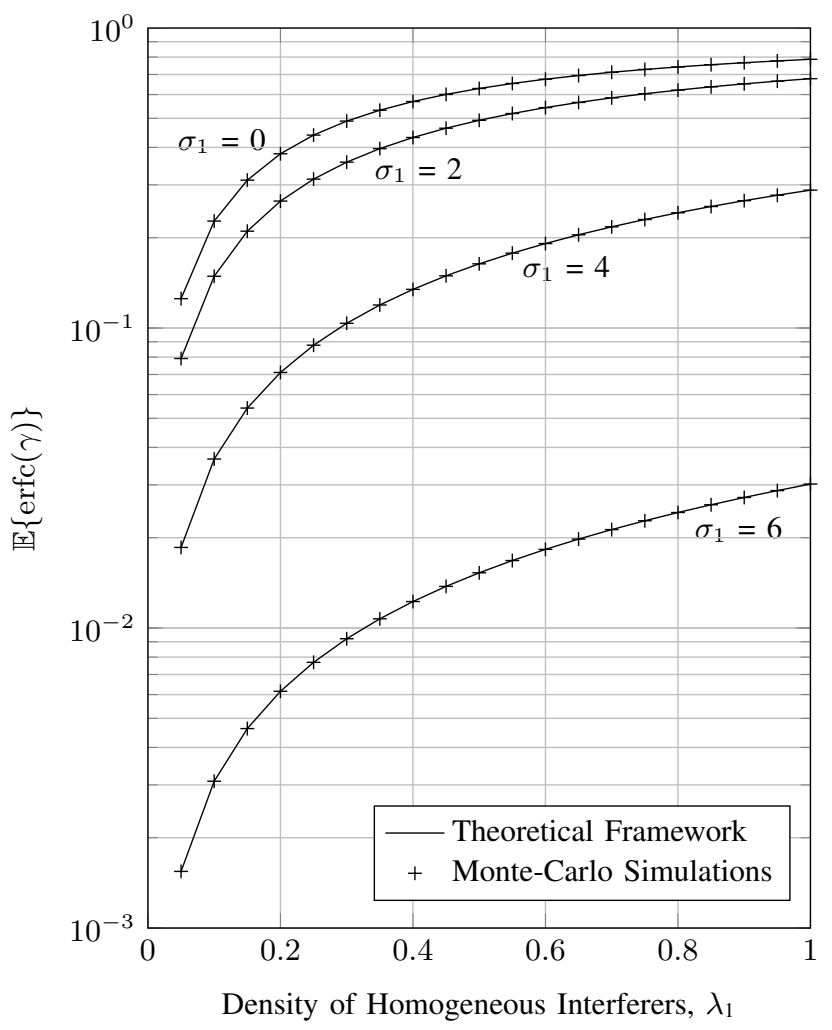

Fig. 3: Average of the complimentary error function of the arbitrary transceiver SINR experiencing different interference volumes. System parameters are: $m=1, \sigma=0, \eta=0, \beta=4, T=1, m_{1}=1$, $r_{e}=0$.

\section{A. Monte-Carlo Simulations}

The methodology used for carrying out the Monte-Carlo simulations in the generalized HetNet scenario is presented below.

1) Set the number of tiers T, PPP deployment densities $\lambda_{t}$, transmit powers $p_{t}$, Nakagami-m fading parameters $m_{t}$, Log-Normal shadowing means $\mu_{t}$ and standard deviations $\sigma_{t}$, path-loss exponent $\beta$, exclusion region radius $r_{e}$, and noise power $\eta$.

2) Define a large circle of radius $D\left(\gg r_{e}\right)$ around a reference receiver located at the origin.

3) Generate the statistical number of tier- $t$ sources $\mathcal{N}_{t}$ using Poisson distribution with average $\pi d^{2} \lambda_{t}$. Then, deploy uniformly-distributed nodes in the bounded region of area $\pi\left(D^{2}-r_{e}^{2}\right)$.

4) Generate independent fading and shadowing channel power gains from all sources.

5) Associate the reference receiver to the closest transmitter of a tier $t^{*}$ which provides the strongest received signal power. Hence, the intended signal power $X_{0}$ can be evaluated.

6) Calculate the aggregate network interference $I_{a g g}$ the reference receiver experiences.

7) Evaluate the instantaneous SINR and rate (nat/s/Hz) of the reference receiver using $\gamma_{t^{*}}=\frac{X_{0}}{I_{a g g}+\eta}$ and $\log \left(1+\gamma_{t^{*}}\right)$, respectively.

8) Compute the corresponding average SINR and rate by

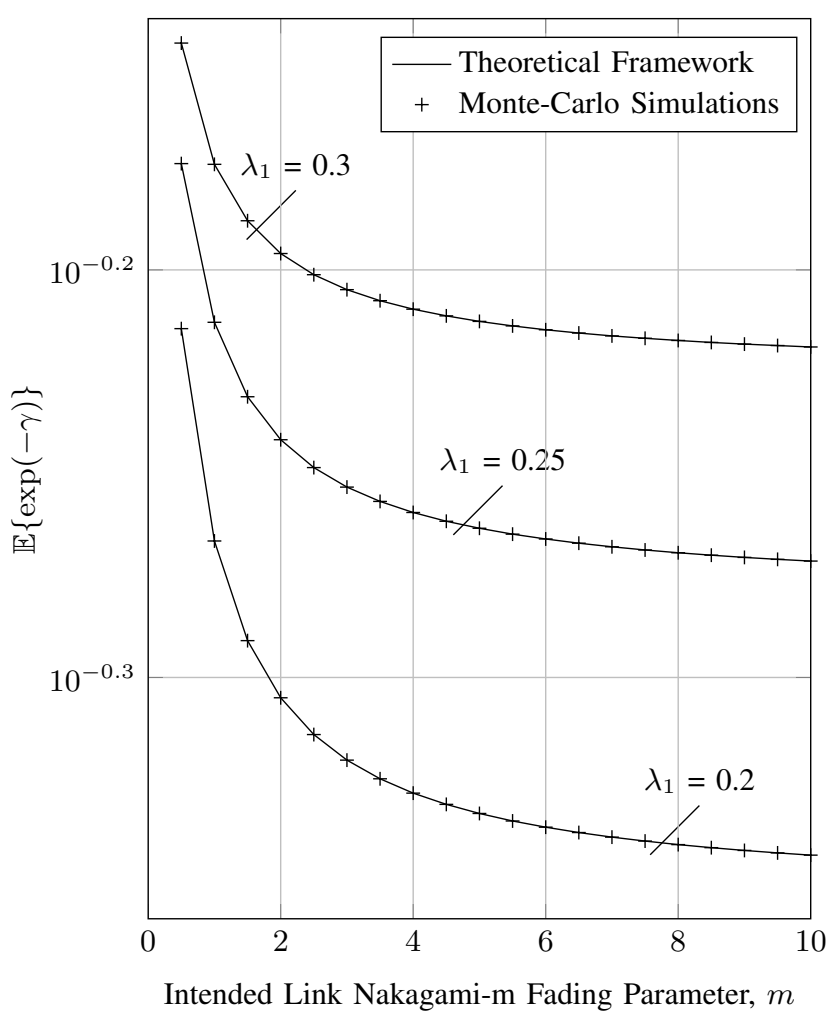

Fig. 4: Average of the exponential function of the arbitrary transceiver SINR over different intended fading channel characteristics. System parameters are: $\sigma=0, \eta=0, \beta=4, T=1, m_{1}=1, \sigma_{1}=0$, $r_{e}=0$.

repeating the steps 3-7 a sufficiently large number of times and then taking the means.

Let us elaborate on the resource-intensity of the theoretical and simulation studies based on a standard workstation at present time. A typical curve using the proposed framework was obtained in the order of minutes whereas the MonteCarlo simulations which were repeated 100k times over a radius of $20 \mathrm{~km}$ required tens of hours to complete. Smaller path-loss exponents, increased densities, and adding tiers, all significantly increase the complexity of the Monte-Carlo trials.

\section{B. Framework Validation}

The curves on Fig. 1 correspond to the average SINR of a typical transceiver around a Poisson field of homogeneous interferers with different densities and path-loss exponents. The impact of a wide range of noise values on average SINR considering two different sparse and dense deployment densities is depicted in Fig. 2. It is shown that the results from our theoretical framework very accurately match those from the Monte-Carlo simulations. The results are otherwise fairly intuitive as smaller PPP-based interference mean, greater path-loss exponent, and higher SNR values all improve the average SINR performance. It can however be noted from Fig. 2 that in noise-dominant operating regimes $\left(\eta \gg I_{a g g}\right)$, the deployment density of interfering sources has negligible impact on performance. 


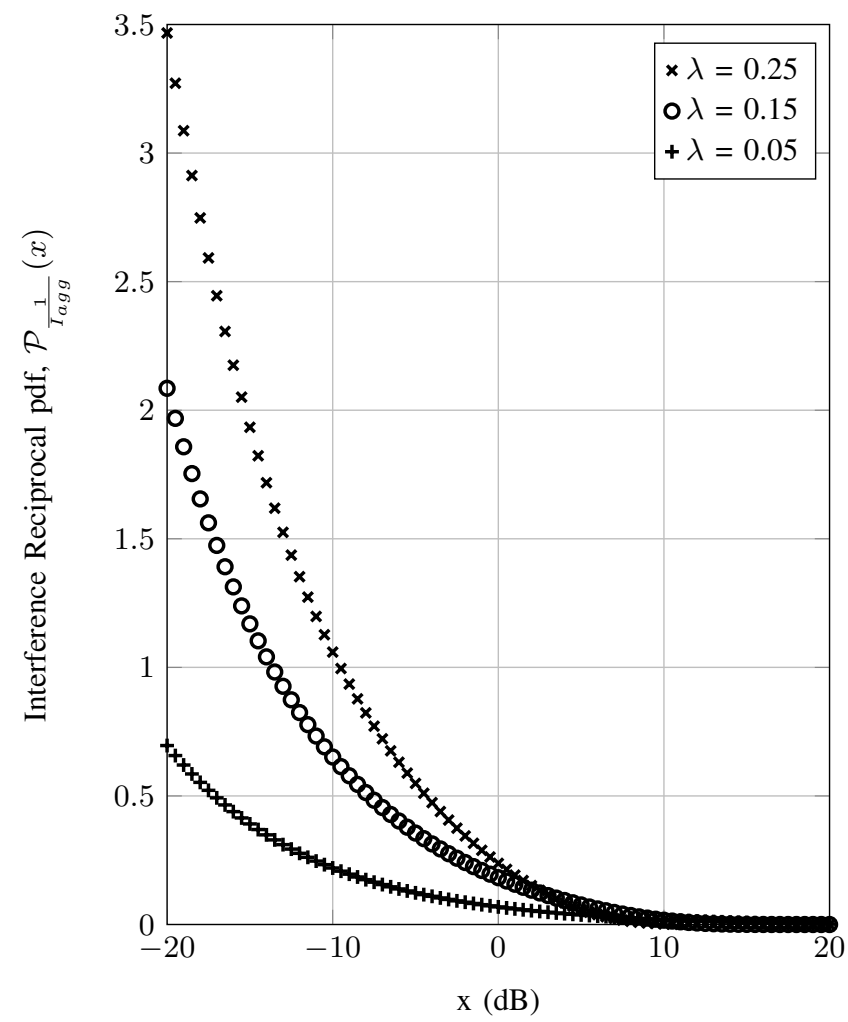

Fig. 5: Density function of the reciprocal of the aggregate interference from a Poisson field of homogeneous interferers. System parameters are: $\eta=0, \beta=4, T=1, m_{1}=1, \sigma_{1}=0, r_{e}=0$.

\section{Error Rate Performance Analysis}

Fig. 3 shows the average of the complimentary error function of SINR over Rayleigh faded and Log-Normal shadowed interfering links considering different PPP-based deployment densities and shadowing variances. It can be observed that with more severe shadowing interfering links, $\mathbb{E}\{\operatorname{erfc}(\gamma)\}$ accordingly decreases, indicating an improved error rate performance. In addition, the exponential function of the SINR for a wide range of intended link fading intensities is plotted in Fig. 4. The figure illustrates that $\mathbb{E}\{\exp (-\gamma)\}$ decreases as $m$ moves away from $\frac{1}{2}$. The gain in error rate performance is however less significant for higher $m$. Improvement in transmission can be achieved via lowering the spatial density of the interferers. Note that the findings from the theoretical and simulation studies match-up closely. Moreover, the impact of interferers density and intended link fading severity on the pdfs of the interference reciprocal and SIR in interferencelimited environment are respectively depicted in Fig. 5 and Fig. 6.

\section{Heterogeneous Cellular Network}

In this part, we focus on examining the performance of a stochastic geometry-based multi-tier cellular network overlaid with PPP-based Micro, Pico, and Femto cells which differ in terms of their operating parameters. In particular, we utilize the practical power model values in [39].

The theoretical average SINR performance of a typical user in the HetNet paradigm considering different exclusion

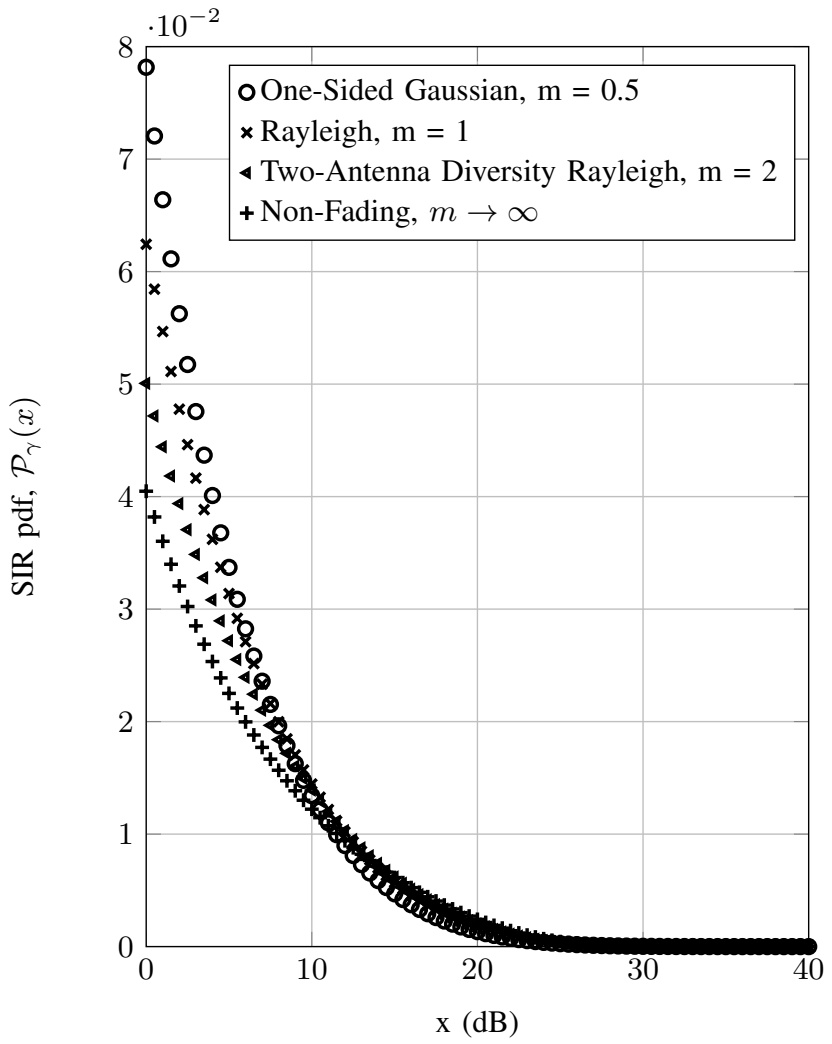

Fig. 6: Density function of the arbitrary transceiver SIR around a Poisson field of homogeneous interferers. System parameters are: $\eta=$ $0, \beta=4, T=1, \lambda_{1}=0.1, m_{1}=0.5, \sigma_{1}=3, r_{e}=0$.

zone radius and SNR values is depicted in Fig. 7. It can be observed that increasing the minimum allowed distance between the transmitter-receiver pair has a significant impact on the achievable average SINR. Mathematically, $\mathbb{E}\{\gamma\} \rightarrow \infty$ as $r_{e} \rightarrow 0$ and $\mathbb{E}\{\gamma\} \rightarrow 0$ as $r_{e} \rightarrow \infty$. Furthermore, decreasing the SNR lowers the average SINR that a typical user experiences; with the difference in performance of noiselimited cases being smaller. In addition, the impact of different path-loss exponents for all intended and interfering links is studied in Fig. 8 where the average SINR of individual tiers in the HetNet is plotted. Lower path-loss exponents significantly degrade the average SINR performance almost irrespective of the noise value selected. SNR, however, plays an important role for higher values of $\beta$, in particular, the lower-power Femto and Pico BSs achieve better results in interferencedominant scenarios.

Next, we study the impact of network densification using small-cell solution on the HetNet average SINR and rate performance in Fig. 9. An important trend is observed in which deploying more Femto-cells increases the average rate at the cost of reduced average SINR. A similar trade-off between the attainable average SINR and rate can be seen in Fig. 10 where the performance is evaluated for different Pico-cells transmit powers. The is because the universal frequency reuse characteristic in the HetNet topology maximizes the spatial reuse and hence spectral efficiency; this however comes at the cost of lower SINR values due to high interference. 


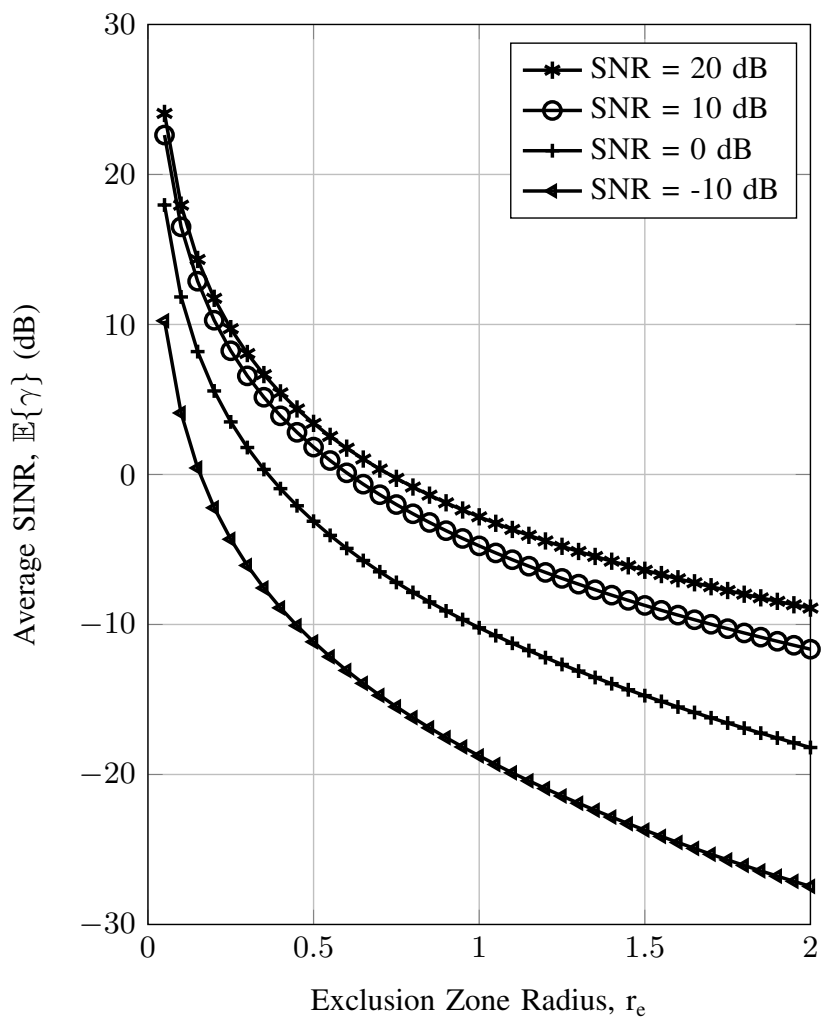

Fig. 7: HetNet average SINR performance with different exclusion zone radius and noise values. System parameters are: $m=1, \beta=4$, $T=3,\left[\lambda_{1}, \lambda_{2}, \lambda_{3}\right]=[0.05,0.1,0.2],\left[p_{1}, p_{2}, p_{3}\right]=[6.3,0.13,0.05]$ $\mathrm{W}, m_{t}=1, t \in \mathcal{T}$

\section{SUMmARY AND CONCLUSIONS}

We have presented a unified analytical framework for evaluating the exact statistics of SINR where interfering sources are spatially-distributed according to the PPP-based abstraction model. The non-direct MGF methodology offers increased analytical tractability and computational efficiency over the conventional direct-pdf approach. In certain cases, closedform solutions for the moments, special functions, and pdf of SINR are developed. As an application example, the proposed methodology is used to characterize the average SINR and rate performance in multi-tier cellular networks over Nakagami$\mathrm{m}$ fading channels with interference exclusion region. The validity of the theoretical findings is confirmed via MonteCarlo simulations for various system setups. Our findings reveal several important trends; in particular we show that with the rapid deployment of small-cells, there exits a trade-off between the attainable average SINR and rate.

\section{APPENDIX A \\ SINR MOMENTS}

Consider a random variable $Y=\frac{X}{I+C}$ where $X$ and $I$ are arbitrary non-negative random variables and $C$ is a constant. Next, we utilize the identity for the $n$-th negative power of $I+C$ [40, eq. (3.381.4)]:

$$
\frac{1}{(I+C)^{n}}=\int_{0}^{+\infty} \frac{z^{n-1}}{\Gamma(n)} \exp (-z(I+C)) \mathrm{d} z .
$$

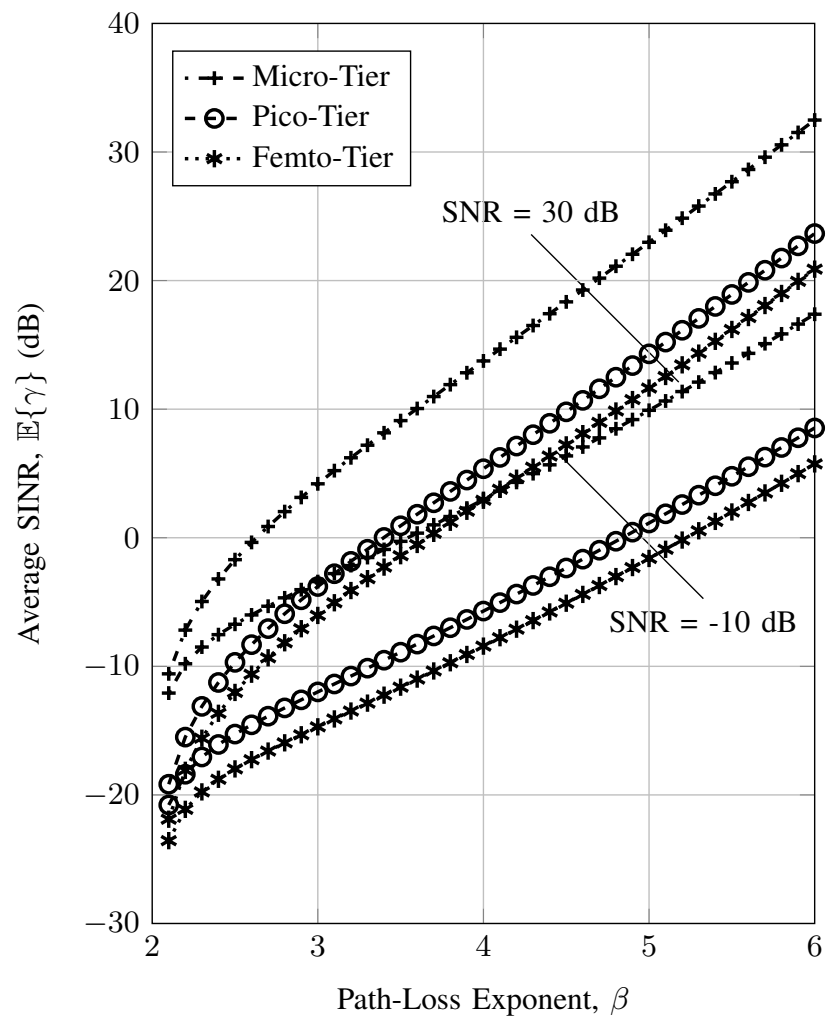

Fig. 8: Effect of path-loss and noise on HetNet individual tiers average SINR performance. System parameters are: $m=1, r_{e}=0.1$, $T=3,\left[\lambda_{1}, \lambda_{2}, \lambda_{3}\right]=[0.05,0.3,0.4],\left[p_{1}, p_{2}, p_{3}\right]=[6.3,0.13,0.05]$ $\mathrm{W}, m_{t}=1, t \in \mathcal{T}$.

The average of the above expression can then be written as

$$
\mathbb{E}\left\{\frac{1}{(I+C)^{n}}\right\}=\int_{0}^{+\infty} \frac{z^{n-1}}{\Gamma(n)} \mathbb{E}\{\exp (-z I)\} \exp (-z C) \mathrm{d} z .
$$

Hence, if $X$ and $I$ are independent, the following expression for the high-order moments of $Y$ holds [27, eq. (5)]:

$\mathbb{E}\left\{\left(\frac{X}{I+C}\right)^{n}\right\}=\mathbb{E}\left\{X^{n}\right\} \int_{0}^{+\infty} \frac{z^{n-1}}{\Gamma(n)} \mathcal{M}_{I}(z) \exp (-z C) \mathrm{d} z$.

\section{APPENDIX B}

\section{INTENDED SIGNAL MOMENTS}

With independent Nakagami-m fading and Log-Normal shadowing channel gains, we have

$$
\begin{aligned}
\mathbb{E}\left\{X_{0}^{n}\right\} & =\mathbb{E}\left\{\left(g_{0} \psi_{0}\right)^{n}\right\}=\mathbb{E}_{\psi_{0}}\left\{\mathbb{E}_{g_{0}}\left\{X_{0}^{n} \mid \psi_{0}\right\}\right\} \\
& =\mathbb{E}_{\psi_{0}}\left\{\frac{\psi_{0}^{n}}{\Gamma(m)}\left(\frac{m}{\Omega}\right)^{m} \int_{0}^{+\infty} g^{n+m-1} \exp \left(-\frac{m g}{\Omega}\right) \mathrm{d} g\right\} \\
& =\frac{\Gamma(n+m)}{\Gamma(m)}\left(\frac{\Omega}{m}\right)^{n} \mathbb{E}_{\psi_{0}}\left\{\psi_{0}^{n}\right\} \\
& =\frac{\Gamma(n+m)}{\Gamma(m)}\left(\frac{\Omega}{m}\right)^{n} \frac{1}{\sqrt{2 \pi} \sigma} \int_{0}^{+\infty} \psi^{n-1} \\
& \times \exp \left(-\frac{(\log (\psi)-\mu)^{2}}{2 \sigma^{2}}\right) \mathrm{d} \psi
\end{aligned}
$$




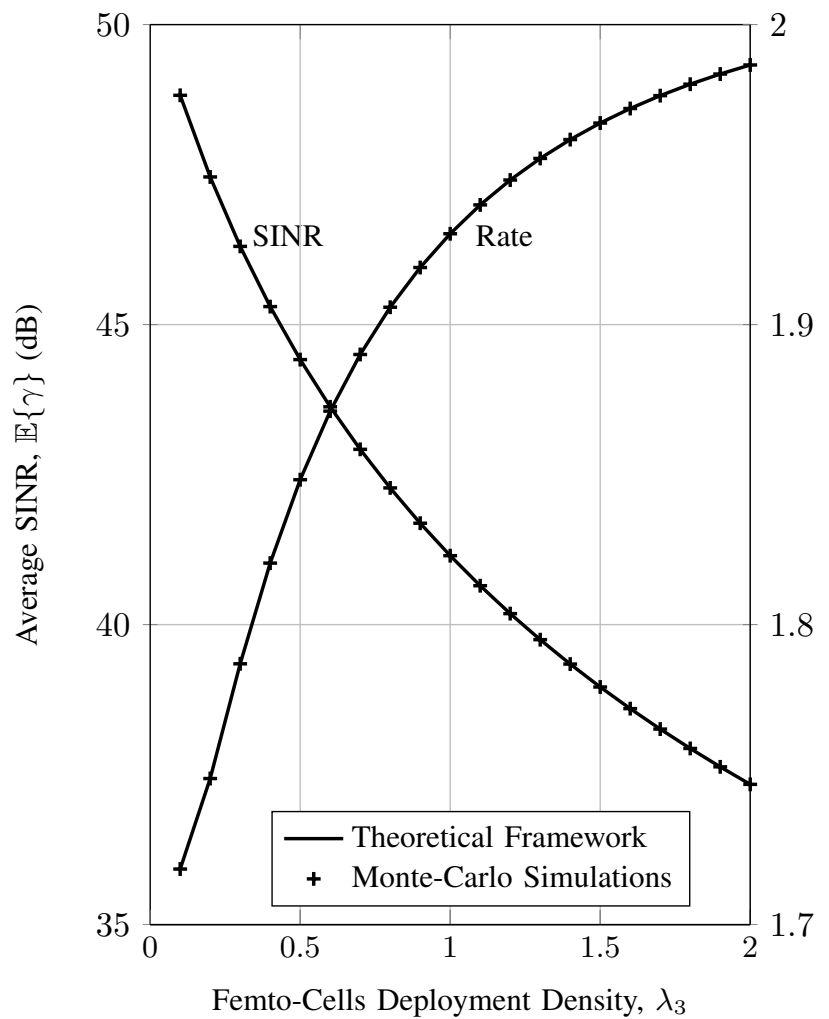

Fig. 9: Impact of Femto-cells deployment density on HetNet SINR and rate performance. System parameters are: $\frac{1}{\eta}=25 \mathrm{~dB}, m=1, r_{e}$ $=0.004, T=3,\left[\lambda_{1}, \lambda_{2}\right]=[0.03,0.05],\left[p_{1}, p_{2}, p_{3}\right]=[6.3,0.13,0.05]$ $\mathrm{W}, m_{t}=1, t \in \mathcal{T}$.

$$
=\frac{\Gamma(n+m)}{\Gamma(m)}\left(\frac{\Omega}{m}\right)^{n} \exp \left(\frac{1}{2}(n \sigma)^{2}+\mu n\right) .
$$

Hence, the average of the intended signal power can be computed by $\mathbb{E}\left\{X_{0}\right\}=\Omega \exp \left(\mu+\frac{\sigma^{2}}{2}\right)$.

\section{APPENDIX C}

\section{PPP-BASED INTERFERENCE STATISTICS}

We consider the interference generated by heterogeneous interferers in a disc of radius $D$ around the reference receiver, and will ultimately take the limit as $D \rightarrow+\infty$. Hence, (C.1). Given interferers are uniformly-distributed, the pdf of their distance to the reference receiver is

$$
\mathcal{P}_{r_{t, k}}(x)= \begin{cases}\frac{2 x}{D^{2}} & 0<x<D \\ 0 & \text { elsewhere }\end{cases}
$$

Let $\mathcal{N}_{t}$ denote the random number of interfering tier- $t$ nodes, thus, (C.1) conditioned on $\mathcal{N}_{t}$ is (C.3) where $g_{t, 1}, \psi_{t, 1}$, and $r_{t, 1}$ represent the fading gain, shadowing gain, and relative distance to victim receiver of an arbitrary tier- $t$ source. By characterizing $\mathcal{N}_{t}$ with a Binomial distribution such that $\mathcal{N}_{t} \sim$ $B\left(\kappa_{t}, \rho_{t}\right)$, we can write (C.4). To proceed, we present the following integral identity (with $\beta>2$ )

$$
\begin{aligned}
\mathbb{E}_{r}\left\{\exp \left(-\alpha r^{-\beta}\right)\right\} & =\int_{0}^{D} \exp \left(-\alpha x^{-\beta}\right) \frac{2 x}{D^{2}} \mathrm{~d} x \\
& =\frac{2}{\beta} \mathscr{E}_{1+\frac{2}{\beta}}\left(\alpha D^{-\beta}\right) .
\end{aligned}
$$

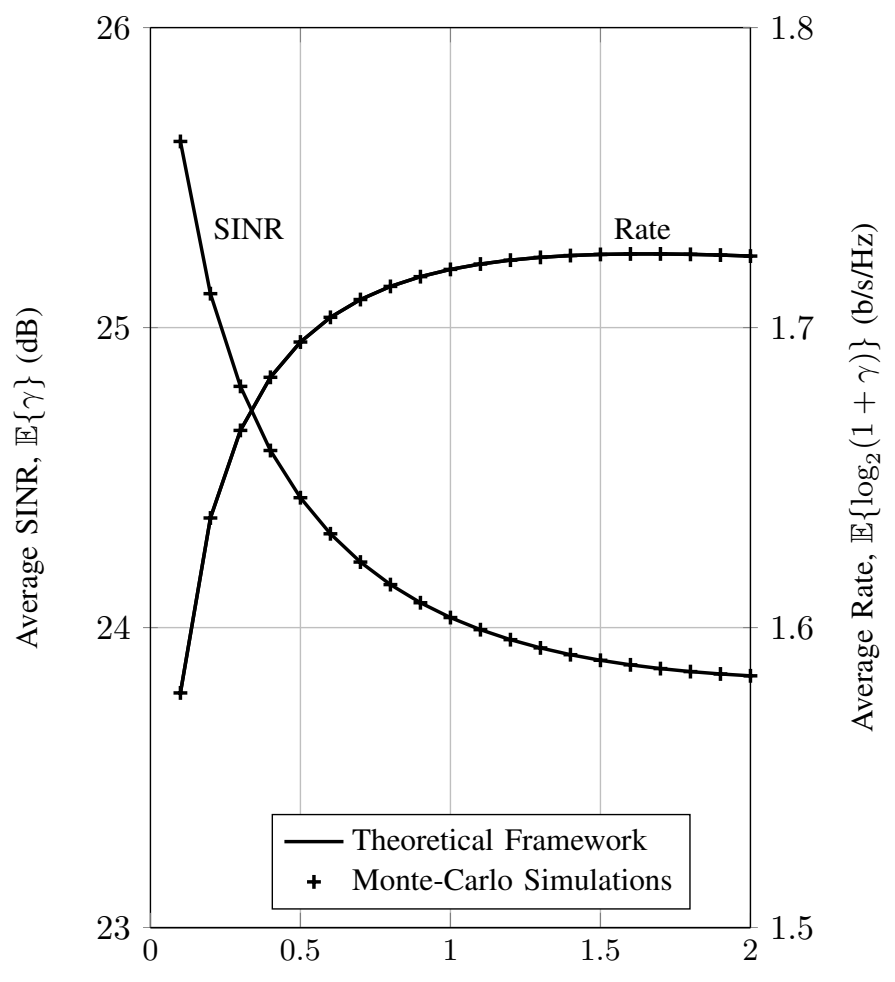

Pico-Cells Transmit Power, $p_{2}(\mathrm{~W})$

Fig. 10: Impact of Pico-cells transmit power on HetNet SINR and rate performance. System parameters are: $\frac{1}{\eta}=20 \mathrm{~dB}, m=2, r_{e}=$ $0.05, T=3,\left[\lambda_{1}, \lambda_{2}, \lambda_{3}\right]=[0.02,0.08,0.1],\left[p_{1}, p_{3}\right]=[6.3,0.05] \mathrm{W}$, $m_{t}=2, t \in \mathcal{T}$.

We continue with the expectation inside (C.4) by first conditioning on $g_{t, 1}$ and $\psi_{t, 1}$ and then taking the average with respect to $r_{t, 1}$ as in (C.6). Now, using (C.6), taking the limits as $D \rightarrow+\infty, \kappa_{t} \rightarrow+\infty, \rho_{t} \rightarrow 0$, and utilizing the Poisson limit theorem with $\frac{\kappa_{t} \rho_{t}}{\pi D^{2}}=\lambda_{t}(<\infty), \mathcal{M}_{I_{a g g}}(z)$ converges to (C.7). Considering Nakagami-m fading channel gains, we take the average of the function of the Gamma-distributed fading channel power gain of the arbitrary interferer as follows

$$
\begin{aligned}
\mathbb{E}_{g_{t, 1}}\left\{g_{t, 1}^{\frac{2}{\beta}}\right\} & =\frac{m_{t}^{m_{t}}}{\Omega_{t}^{m_{t}} \Gamma\left(m_{t}\right)} \int_{0}^{+\infty} g^{\frac{2}{\beta}+m_{t}-1} \exp \left(\frac{-m_{t} g}{\Omega_{t}}\right) \mathrm{d} g \\
& =\left(\frac{\Omega_{t}}{m_{t}}\right)^{\frac{2}{\beta}} \frac{\Gamma\left(m_{t}+\frac{2}{\beta}\right)}{\Gamma\left(m_{t}\right)} .
\end{aligned}
$$

Considering the shadowing power gain of the arbitrary interferer is a Log-Normal random variable with mean $\mu_{t}$ and standard deviation $\sigma_{t}$, we have

$$
\begin{aligned}
\mathbb{E}_{\psi_{t, 1}}\left\{\psi_{t, 1}^{\frac{2}{\beta}}\right\} & =\int_{0}^{+\infty} \frac{\psi^{\frac{2}{\beta}-1}}{\sqrt{2 \pi \sigma_{t}^{2}}} \exp \left(-\frac{\left(\log (\psi)-\mu_{t}\right)^{2}}{2 \sigma_{t}^{2}}\right) \mathrm{d} \psi \\
& =\exp \left(\frac{2}{\beta}\left(\mu_{t}+\frac{\sigma_{t}^{2}}{\beta}\right)\right) .
\end{aligned}
$$

By removing the condition from (C.7) using (C.8) and (C.9), we arrive at Lemma 2. 


$$
\mathcal{M}_{I_{a g g}}(z)=\lim _{D \rightarrow+\infty} \mathbb{E}_{g_{t, k}, \psi_{t, k}, r_{t, k}}\left\{\exp \left(-z \sum_{t \in \mathcal{T}} \sum_{k \in \Phi_{t}} g_{t, k} \psi_{t, k} r_{t, k}^{-\beta}\right)\right\}
$$

$$
\mathcal{M}_{I_{\text {agg }}}\left(z \mid \mathcal{N}_{t}\right)=\lim _{D \rightarrow+\infty} \prod_{t \in \mathcal{T}}\left(\mathbb{E}_{g_{t, 1}, \psi_{t, 1}, r_{t, 1}}\left\{\exp \left(-z g_{t, 1} \psi_{t, 1} r_{t, 1}^{-\beta}\right)\right\}\right)^{\mathcal{N}_{t}}
$$

$$
\mathcal{M}_{I_{\text {agg }}}(z)=\lim _{D \rightarrow+\infty} \prod_{t \in \mathcal{T}}\left(\rho_{t} \mathbb{E}_{g_{t, 1}, \psi_{t, 1}, r_{t, 1}}\left\{\exp \left(-z g_{t, 1} \psi_{t, 1} r_{t, 1}^{-\beta}\right)\right\}+1-\rho_{t}\right)^{\kappa_{t}}
$$

$$
\mathbb{E}_{r_{t, 1}}\left\{\exp \left(-z g_{t, 1} \psi_{t, 1} r_{t, 1}^{-\beta}\right) \mid g_{t, 1}, \psi_{t, 1}\right\}=\frac{2}{\beta} E_{1+\frac{2}{\beta}}\left(z g_{t, 1} \psi_{t, 1} D^{-\beta}\right)
$$

$$
\mathcal{M}_{I_{\text {agg }}}(z)=\prod_{t \in \mathcal{T}} \exp \left(-\pi \lambda_{t} \Gamma\left(1-\frac{2}{\beta}\right) \mathbb{E}_{g_{t, 1}, \psi_{t, 1}}\left\{\left(z g_{t, 1} \psi_{t, 1}\right)^{\frac{2}{\beta}}\right\}\right)
$$

\section{APPENDIX D}

PPP-BASED INTERFERENCE STATISTICS WITH EXCLUSION ZONE

Here, we incorporate the impact of exclusion region on interference characterization using a similar approach to that in Appendix C. As a result, only key differences are described below.

In this case, the pdf of the interferers distance to reference receiver can be written as

$$
\mathcal{P}_{r_{t, k}}(x)= \begin{cases}\frac{2 x}{D^{2}-r_{e}^{2}} & r_{e}<x<D \\ 0 & \text { elsewhere }\end{cases}
$$

Hence, the following integral identity holds (with $\beta>2$ )

$$
\begin{aligned}
\mathbb{E}_{r}\left\{\exp \left(-\alpha r^{-\beta}\right)\right\} & =\int_{r_{e}}^{D} \exp \left(-\alpha x^{-\beta}\right) \frac{2 x}{\left(D-r_{e}\right)^{2}} \mathrm{~d} x \\
& =\frac{\Gamma\left(-\frac{2}{\beta}, \frac{\alpha}{D^{\beta}}\right)-\Gamma\left(-\frac{2}{\beta}, \frac{\alpha}{r_{e}^{\beta}}\right)}{\frac{\beta}{2 \alpha^{\frac{2}{\beta}}}\left(D-r_{e}\right)^{2}} .
\end{aligned}
$$

By taking the underlying limits and applying the Poisson limit theorem, $\mathcal{M}_{I_{\text {agg }}}(z)$ converges to (D.3). Considering Nakagami-m fading channels, we can take the average over $g_{t, 1}$ using (C.8),

$$
\begin{aligned}
& \mathbb{E}_{g_{t, 1}}\left\{g_{t, 1}^{\frac{2}{\beta}} \Gamma\left(1-\frac{2}{\beta}, \alpha g_{t, 1}\right)\right\}=\frac{m_{t}^{m_{t}}}{\Omega_{t}^{m_{t}} \Gamma\left(m_{t}\right)} \int_{0}^{+\infty} g^{m_{t}+\frac{2}{\beta}-1} \\
& \times \exp \left(-\frac{m_{t} g}{\Omega_{t}}\right) \Gamma\left(1-\frac{2}{\beta}, \alpha g\right) \mathrm{d} g=\frac{m_{t}}{\alpha^{\left(m_{t}+\frac{2}{\beta}\right)}\left(m_{t}+\frac{2}{\beta}\right)} \\
& \times\left(\frac{m_{t}}{\Omega_{t}}\right)^{m_{t}}{ }_{2} F_{1}\left(m_{t}+1, m_{t}+\frac{2}{\beta} ; m_{t}+\frac{2}{\beta}+1 ;-\frac{m_{t}}{\alpha \Omega_{t}}\right)
\end{aligned}
$$

and

$$
\begin{aligned}
& \mathbb{E}_{g_{t, 1}}\left\{\exp \left(-\alpha g_{t, 1}\right)\right\}=\frac{m_{t}^{m_{t}}}{\Omega_{t}^{m_{t}} \Gamma\left(m_{t}\right)} \int_{0}^{+\infty} g^{m_{t}-1} \\
& \times \exp \left(-g\left(\alpha+\frac{m_{t}}{\Omega_{t}}\right)\right) \mathrm{d} g=\left(\frac{m_{t}}{\Omega_{t}}\right)^{m_{t}}\left(\alpha+\frac{m}{\omega}\right)^{-m} \\
& =\left(\frac{m_{t}}{m_{t}+\alpha \Omega_{t}}\right)^{m_{t}} .
\end{aligned}
$$

Hence, (39) is obtained.

\section{APPENDIX E}

\section{SINR MOMENTS IN INTERFERENCE-LIMITED} ENVIRONMENT

The following expression is obtained in interference-limited environment (when $\eta \rightarrow 0$ )

$$
\int_{0}^{+\infty} \frac{x^{n-1}}{\Gamma(n)} \exp \left(-\alpha x^{p}\right) \mathrm{d} x=\frac{1}{p} \alpha^{\frac{-n}{p}} \frac{\Gamma\left(\frac{n}{p}\right)}{\Gamma(n)} .
$$

Utilizing the above result together with the closed-form analytical expression of the aggregate interference statistics in Lemma 2, we can obtain (15).

\section{APPENDIX F}

SINR MOMENTS Under ARbitraRy SNRs with $\beta=4$

Considering the effect of non-zero noise variance (i.e., $\eta \neq$ 0 ), with $\beta=4$ we have (F.1). Further, when $n=1$, the integral identity (F.2) holds (with $\eta \neq 0$ ). Hence, we arrive at the result in Lemma 5. 


$$
\begin{aligned}
\mathcal{M}_{I_{\text {agg }}}(z) & =\prod_{t \in \mathcal{T}} \exp \left(-\pi \lambda_{t} \mathbb{E}_{g_{t, 1}}\left\{\left[\Gamma\left(1-\frac{2}{\beta}\right)-\Gamma\left(1-\frac{2}{\beta}, \frac{z g_{t, 1}}{r_{e}^{\beta}}\right)\right]\right.\right. \\
& \left.\left.\times\left(z g_{t, 1}\right)^{\frac{2}{\beta}}+r_{e}^{2}\left(\exp \left(-\frac{z g_{t, 1}}{r_{e}^{\beta}}\right)-1\right)\right\}\right)
\end{aligned}
$$

$$
\int_{0}^{+\infty} \frac{z^{n-1}}{\Gamma(n)} \exp (-\sqrt{z} \mathcal{A}) \exp (-z \eta) \mathrm{d} z=\frac{{ }_{1} F_{1}\left(n ; \frac{1}{2} ; \frac{1}{4 \eta} \mathcal{A}^{2}\right)-\frac{\Gamma\left(n+\frac{1}{2}\right)}{\sqrt{\eta} \Gamma(n)} \mathcal{A}_{1} F_{1}\left(n+\frac{1}{2} ; \frac{3}{2} ; \frac{1}{4 \eta} \mathcal{A}^{2}\right)}{\eta^{n}}
$$

$$
\int_{0}^{+\infty} \exp (-\sqrt{z} \mathcal{A}) \exp (-z \eta) \mathrm{d} z=\frac{1}{\eta}+\frac{1}{2 \eta^{3 / 2}} \sqrt{\pi} \mathcal{A} \exp \left(\frac{1}{4 \eta} \mathcal{A}^{2}\right)\left(\operatorname{erf}\left(\frac{1}{2 \sqrt{\eta}} \mathcal{A}\right)-1\right)
$$

\section{APPENDIX G}

Complimentary Error Function Average with $\eta=0$

$$
\text { AND } \beta=4
$$
sion

Using (20), we can write the following conditional expres-

$$
\begin{aligned}
\mathbb{E}\left\{\operatorname{erfc}(\sqrt{\gamma}) \mid X_{0}\right\} & =1-\frac{2}{\sqrt{\pi}} \sum_{n=0}^{+\infty} \frac{(-1)^{n}}{n !(2 n+1)} \mathbb{E}\left\{\gamma^{n+\frac{1}{2}}\right\} \\
& =1-\frac{4}{\sqrt{\pi}} \sum_{n=0}^{+\infty} \frac{(-1)^{n}}{n !(2 n+1)} \frac{\Gamma(2 n+1)}{\Gamma\left(n+\frac{1}{2}\right)} \frac{X_{0}^{n+\frac{1}{2}}}{\mathcal{A}^{2 n+1}}
\end{aligned}
$$

By utilizing

$$
\Gamma(2 n+1)=\frac{1}{2 \sqrt{\pi}} 2^{2 n+1} \Gamma\left(n+\frac{1}{2}\right) \Gamma(n+1)
$$

we get

$$
\mathbb{E}\left\{\operatorname{erfc}(\sqrt{\gamma}) \mid X_{0}\right\}=1-\frac{2}{\pi} \sum_{n=0}^{+\infty} \frac{(-1)^{n}}{2 n+1} \frac{X_{0}^{n+\frac{1}{2}}}{\left(\frac{1}{2} \mathcal{A}\right)^{2 n+1}} .
$$

Finally, recognizing the series

$$
\arctan \left(\frac{1}{x}\right)=\sum_{k=0}^{+\infty} \frac{(-1)^{k}}{2 k+1} \frac{1}{x^{2 k+1}}
$$

we arrive at (21). Next, consider the integral identity in (G.5) for a positive Gamma-distributed random variable $x$. Hence, in the case of Nakagami-m fading for the intended link, we can obtain (22).

\section{APPENDIX H}

EXPONENTIAL FUNCTION AVERAGE WITH $\eta=0$ AND

$$
\beta=4
$$

Utilizing (24), we can derive the following conditional expression

$$
\begin{aligned}
& \mathbb{E}\left\{\exp (-\gamma) \mid X_{0}\right\}=1+\sum_{n=1}^{+\infty} \frac{(-1)^{n} \Gamma(2 n+1)}{n ! \Gamma(n+1)} \frac{X_{0}^{n}}{\mathcal{A}^{2 n}} \\
& =\frac{1}{\sqrt{\pi}} \sum_{n=0}^{+\infty}\left(\frac{-4 X_{0}}{\mathcal{A}^{2}}\right)^{n} \frac{\Gamma\left(n+\frac{1}{2}\right)}{\Gamma(n+1)}
\end{aligned}
$$

$$
\begin{aligned}
& =1-\frac{2 X_{0}}{\mathcal{A}^{2}} \sum_{n=0}^{\infty} \frac{\left(\frac{-4 X_{0}}{\mathcal{A}^{2}}\right)^{n}}{n !} \frac{\Gamma(2)}{\Gamma(n+2)} \frac{\Gamma\left(n+\frac{3}{2}\right)}{\Gamma\left(\frac{3}{2}\right)} \frac{\Gamma(n+1)}{\Gamma(1)} \\
& =1-\frac{2 X_{0}}{\mathcal{A}^{2}}{ }_{2} F_{1}\left(\frac{3}{2}, 1 ; 2 ; \frac{-4 X_{0}}{\mathcal{A}^{2}}\right)
\end{aligned}
$$

where we have used the functional equations (G.2) and

$$
\Gamma\left(n+\frac{1}{2}\right)=\sqrt{\pi} n !\left(\begin{array}{c}
n-\frac{1}{2} \\
n
\end{array}\right) .
$$

Next, we can verify that

$$
\begin{aligned}
{ }_{2} F_{1}\left(\frac{3}{2}, 1 ; 2 ; \frac{-4 X_{0}}{\mathcal{A}^{2}}\right) & =\int_{0}^{1}\left(1+x \frac{4 X_{0}}{\mathcal{A}^{2}}\right)^{-\frac{3}{2}} \mathrm{~d} x \\
& =\frac{\mathcal{A}^{2}}{2 X_{0}}\left(1-\frac{1}{\sqrt{1+\frac{4 X_{0}}{\mathcal{A}^{2}}}}\right) .
\end{aligned}
$$

Substituting (H.3) in (H.1) results in (25).

We can proceed further by utilizing the following integral identity for a positive random variable $x$ following a Gamma distribution (with $\alpha>0$ and $m>0$ )

$$
\begin{aligned}
& \mathbb{E}_{x}\left\{\frac{1}{\sqrt{1+\alpha x}}\right\}=\int_{0}^{+\infty} \frac{m^{m}}{\Gamma(m) \sqrt{1+\alpha g}} g^{m-1} \exp (-m g) \mathrm{d} g \\
& =\frac{\sqrt{\pi} m^{m}}{\alpha^{m} \cos (\pi m)}{ }_{1} \tilde{F}_{1}\left(m ; m+\frac{1}{2} ; \frac{m}{\alpha}\right)-\frac{\pi \sqrt{m}}{\sqrt{\alpha} \Gamma(m) \cos (\pi m)} \\
& \times{ }_{1} \tilde{F}_{1}\left(\frac{1}{2} ; \frac{3}{2}-m ; \frac{m}{\alpha}\right) .
\end{aligned}
$$

Hence, in the case of Nakagami-m faded intended link, we arrive at (26).

\section{APPENDIX I}

\section{DENSITY FUNCTION OF SIR WITH $\beta=4$}

The density function of a continuous random variable $Y$ with $\operatorname{MGF} \mathcal{M}_{Y}(s)=\mathbb{E}\{\exp (s Y)\}=$ $\int_{-\infty}^{+\infty} \mathcal{P}_{Y}(x) \exp (s x) \mathrm{d} x$ can be expressed as

$\mathcal{P}_{Y}(x)=\mathcal{L}^{-1}\left(\mathcal{M}_{Y}(s)\right)=\frac{1}{2 \pi i} \int_{c-i_{-\infty}}^{c+i_{+\infty}} \mathcal{M}_{Y}(s) \exp (-s x) \mathrm{d} s$ 


$$
\begin{aligned}
\mathbb{E}_{x}\{\arctan (\sqrt{x})\} & =\int_{0}^{+\infty} \frac{m^{m}}{\Gamma(m)} g^{m-1} \exp (-m g) \arctan (\sqrt{g}) \mathrm{d} g \\
& =\frac{\pi}{2}\left(1+\frac{\left(\sqrt{\pi}(-1)^{m} \sqrt{m}_{2} \tilde{F}_{2}\left(\frac{1}{2}, 1 ; \frac{3}{2}, \frac{3}{2}-m ; m\right)-\Gamma(m)+\Gamma(m,-m)\right)}{(-1)^{m} \Gamma(m) \cos (\pi m)}\right)
\end{aligned}
$$

$$
\mathbb{E}_{x}\left\{\frac{1}{\sqrt{x}} \exp \left(-\frac{\alpha}{x}\right)\right\}=\int_{0}^{+\infty} \frac{m^{m}}{\Gamma(m)} g^{m-\frac{3}{2}} \exp \left(-\left(\frac{\alpha}{g}+g m\right)\right) \mathrm{d} g=\frac{2 m^{\frac{m}{2}+\frac{1}{4}}}{\Gamma(m)} \alpha^{\frac{m}{2}-\frac{1}{4}} K_{\frac{1}{2}-m}(2 \sqrt{m \alpha})
$$

where $\mathcal{L}^{-1}($.$) denotes the inverse Laplace operation and c$ is an arbitrary constant. It is generally very difficult to directly obtain $\mathcal{P}_{Y}(x)$ using the above expression. One can however recognize that the average exponential function developed in (25) can be alternatively obtained via Laplace transformation of a certain function. Specifically, consider the following integral identity

$$
\int_{0}^{+\infty} \frac{1}{\sqrt{\pi \alpha x}} \exp \left(-\frac{x}{\alpha}\right) \exp (-z x) \mathrm{d} x=\frac{1}{\sqrt{\alpha z+1}} .
$$

As a result, the pdf of the SIR conditioned on the useful signal can be recovered as

$$
\begin{aligned}
\mathcal{P}_{\gamma}\left(x \mid X_{0}\right) & =\mathcal{L}^{-1}\left(\mathcal{M}_{\gamma}\left(z \mid X_{0}\right)\right)=\mathcal{L}^{-1}\left(\frac{\mathcal{A}}{\sqrt{\mathcal{A}^{2}+4 X_{0} z}}\right) \\
& =\frac{\mathcal{A}}{2 \sqrt{\pi X_{0} x}} \exp \left(-\frac{\mathcal{A}^{2} x}{4 X_{0}}\right) .
\end{aligned}
$$

The above implies that the aggregate interference reciprocal pdf has a closed-form given in (28).

In order to derive a closed-form expression for the pdf of SIR, consider the integral identity (I.4) for a positive Gammadistributed random variable (with $\alpha>0$ and $m>0$ ). The pdf of SIR under intended Nakagami-m fading can therefore be expressed as in (29).

\section{REFERENCES}

[1] M. Haenggi, J. Andrews, F. Baccelli, O. Dousse, and M. Franceschetti, "Stochastic geometry and random graphs for the analysis and design of wireless networks," IEEE J. Sel. Areas Commun., vol. 27, no. 7, pp. 1029-1046, Sep. 2009.

[2] K. Hamdi, "Exact SINR analysis of wireless OFDM in the presence of carrier frequency offset," IEEE Trans. Wireless Commun., vol. 9, no. 3, pp. 975-979, Mar. 2010.

[3] B. Hagerman, "Downlink relative co-channel interference powers in cellular radio systems," in Vehicular Technology Conference, 1995 IEEE 45th, vol. 1, Jul. 1995, pp. 366-370.

[4] A. Wyner, "Shannon-theoretic approach to a Gaussian cellular multiple-access channel," IEEE Trans. Inf. Theory, vol. 40, no. 6, pp. 1713-1727, Nov. 1994.

[5] E. Sousa and J. Silvester, "Optimum transmission ranges in a direct-sequence spread-spectrum multihop packet radio network," IEEE J. Sel. Areas Commun., vol. 8, no. 5, pp. 762-771, Jun. 1990.

[6] F. Baccelli, M. Klein, M. Lebourges, and S. Zuyev, "Stochastic geometry and architecture of communication networks," Telecommunication Systems, vol. 7, no. 1-3, pp. 209-227, 1997.

[7] H. Elsawy, E. Hossain, and M. Haenggi, "Stochastic geometry for modeling, analysis, and design of multi-tier and cognitive cellular wireless networks: A survey," IEEE Commun. Surveys Tuts., vol. 15, no. 3, pp. 996-1019, Jun. 2013.

[8] F. Yilmaz and M.-S. Alouini, "A unified MGF-based capacity analysis of diversity combiners over generalized fading channels," IEEE Trans. Commun., vol. 60, no. 3, pp. 862-875, Mar. 2012.

[9] M. Di Renzo, A. Guidotti, and G. Corazza, "Average rate of downlink heterogeneous cellular networks over generalized fading channels: A stochastic geometry approach,” IEEE Trans. Commun., vol. 61, no. 7, pp. 3050-3071, Jul. 2013.
[10] H. Dhillon, R. Ganti, F. Baccelli, and J. Andrews, "Modeling and analysis of K-tier downlink heterogeneous cellular networks," IEEE J. Sel. Areas Commun., vol. 30, no. 3, pp. 550-560, Apr. 2012.

[11] H. Dhillon, R. Ganti, and J. Andrews, "Load-aware modeling and analysis of heterogeneous cellular networks," IEEE Trans. Wireless Commun., vol. 12, no. 4 pp. 1666-1677, Apr. 2013.

[12] M. Di Renzo, F. Graziosi, and F. Santucci, "Channel capacity over generalized fading channels: A novel MGF-based approach for performance analysis and design of wireless communication systems," IEEE Trans. Veh. Technol., vol. 59, no. 1, pp. 127-149, Jan. 2010

[13] M. K. Simon and M.-S. Alouini, Digital communication over fading channels, ser. Wiley series in telecommunications and signal processing. Hoboken, N.J. Wiley-Interscience, 2005.

[14] A. Shojaeifard, K. Hamdi, E. Alsusa, D. So, and J. Tang, "A unified model for the design and analysis of spatially-correlated load-aware HetNets," IEEE Trans. Commun., vol. 62, no. 11, pp. 4110-4125, Nov. 2014.

[15] M. Win, P. Pinto, and L. Shepp, "A mathematical theory of network interference and its applications," Proc. IEEE, vol. 97, no. 2, pp. 205-230, Feb. 2009.

[16] G. Farhadi and N. Beaulieu, "On the ergodic capacity of multi-hop wireless relaying systems," IEEE Trans. Wireless Commun., vol. 8, no. 5, pp. 2286-2291, May 2009.

[17] R. Ganti, F. Baccelli, and J. Andrews, "Series expansion for interference in wireless networks," IEEE Trans. Inform. Theory, vol. 58, no. 4, pp. 2194-2205, Apr. 2012.

[18] C. Zhong, M. Matthaiou, G. Karagiannidis, and T. Ratnarajah, "Generic ergodic capacity bounds for fixed-gain af dual-hop relaying systems," IEEE Trans. Veh. Technol., vol. 60, no. 8, pp. 3814-3824, Oct. 2011.

[19] O. Waqar, D. McLernon, and M. Ghogho, "Exact evaluation of ergodic capacity for multihop variable-gain relay networks: A unified framework for generalized fading channels," IEEE Trans. Veh. Technol., vol. 59, no. 8, pp. 4181-4187, Oct. 2010 .

[20] B. Blaszczyszyn and H. P. Keeler, "Studying the SINR process of the typical user in poisson networks by using its factorial moment measures," CoRR, vol. abs/1401.4005, 2014.

[21] A. Guo and M. Haenggi, "Spatial stochastic models and metrics for the structure of base stations in cellular networks," IEEE Trans. Wireless Commun., vol. 12 no. 11 , pp. 5800-5812, Nov. 2013.

[22] P. Madhusudhanan, J. Restrepo, Y. Liu, T. Brown, and K. Baker, "Downlink performance analysis for a generalized shotgun cellular system," IEEE Trans. Wireless Commun., vol. 13, no. 12, pp. 6684-6696, Dec. 2014.

[23] H. Dhillon, M. Kountouris, and J. Andrews, "Downlink MIMO hetnets: Modeling, ordering results and performance analysis," IEEE Trans. Wireless Commun., vol. 12, no. 10 , pp. 5208-5222, Oct. 2013.

[24] R. Tanbourgi, H. Dhillon, J. Andrews, and F. Jondral, "Effect of spatial interference correlation on the performance of maximum ratio combining," IEEE Trans. Wireless Commun., vol. 13, no. 6, pp. 3307-3316, Jun. 2014.

[25] R. Tanbourgi, S. Singh, J. Andrews, and F. Jondral, "A tractable model for noncoherent joint-transmission base station cooperation," IEEE Trans. Wireless Commun., vol. 13, no. 9, pp. 4959-4973, Sep. 2014.

[26] H. Inaltekin, M. Chiang, H. Poor, and S. Wicker, "On unbounded path-loss models: effects of singularity on wireless network performance," IEEE J. Sel. Areas Commun., vol. 27, no. 7, pp. 1078-1092, Sep. 2009.

[27] K. Hamdi, "On the statistics of signal-to-interference plus noise ratio in wireless communications," IEEE Trans. Commun., vol. 57, no. 11, pp. 3199-3204, Nov. 2009.

[28] Y. Shobowale and K. Hamdi, "A unified model for interference analysis in unlicensed frequency bands," IEEE Trans. Wireless Commun., vol. 8, no. 8, pp. 4004-4013, Aug. 2009.

[29] U. Schilcher, C. Bettstetter, and G. Brandner, "Temporal correlation of interference in wireless networks with rayleigh block fading," IEEE Trans. Mobile Comput., vol. 11 , no. 12 , pp. $2109-2120$, Dec. 2012.

[30] A. Crismani, S. Toumpis, U. Schilcher, G. Brandner, and C. Bettstetter, "Cooperative relaying under spatially and temporally correlated interference," IEEE Trans. Veh. Technol., to be published.

[31] M. Abramowitz and I. A. Stegun, Handbook of Mathematical Functions with Formulas, Graphs, and Mathematical Tables. New York: Dover, 1964.

[32] N. Ermolova, "Analysis of OFDM error rates over nonlinear fading radio channels," IEEE Trans. Wireless Commun., vol. 9, no. 6, pp. 1855-1860, Jun. 2010. 
[33] F. Yilmaz and M.-S. Alouini, "Novel asymptotic results on the high-order statistics of the channel capacity over generalized fading channels," in Signal Processing Advances in Wireless Communications (SPAWC), 2012 IEEE 13th International Workshop on, Jun. 2012, pp. 389-393.

[34] M. Haenggi and R. K. Ganti, "Interference in large wireless networks," Foundations and Trends in Networking, vol. 3, no. 2, pp. 127-248, 2009.

[35] S. Singh, H. Dhillon, and J. Andrews, "Offloading in heterogeneous networks: Modeling, analysis, and design insights," IEEE Trans. Wireless Commun., vol. 12, no. 5, pp. 2484-2497, May 2013.

[36] H. Keeler, B. Blaszczyszyn, and M. Karray, "SINR-based K-coverage probability in cellular networks with arbitrary shadowing," in Information Theory Proceedings (ISIT), 2013 IEEE International Symposium on, Jul. 2013, pp. 1167-1171.

[37] H. Dhillon and J. Andrews, "Downlink rate distribution in heterogeneous cellular networks under generalized cell selection," IEEE Wireless Commun. Lett., vol. 3, no. 1, pp. 42-45, Feb. 2014.

[38] K. Hamdi, "A useful lemma for capacity analysis of fading interference channels," IEEE Trans. Commun., vol. 58, no. 2, pp. 411-416, Feb. 2010.

[39] G. Auer, V. Giannini, C. Desset, I. Godor, P. Skillermark, M. Olsson, M. Imran, D. Sabella, M. Gonzalez, O. Blume, and A. Fehske, "How much energy is needed to run a wireless network?" IEEE Trans. Wireless Commun., vol. 18, no. 5, pp. 40-49, Oct. 2011.

[40] I. S. Gradshteyn and I. M. Ryzhik, Table of integrals, series, and products, 7th ed. Elsevier/Academic Press, 2007. 